\title{
Experimental study of substrate roughness and surfactant effects on the Landau-Levich law
}

\author{
R. Krechetnikov \\ Division of Engineering and Applied Science, Caltech, Pasadena, California 91125 \\ G. M. Homsy \\ Department of Mechanical Engineering, University of California, Santa Barbara, California 93106
}

(Received 25 November 2004; accepted 2 September 2005; published online 31 October 2005)

\begin{abstract}
In this work we present an experimental study of deviations from the classical Landau-Levich law in the problem of dip coating. Among the examined causes leading to deviations are the nature of the liquid-gas and liquid-solid interfaces. The thickness of the coating film created by withdrawal of a plate from a bath was measured gravimetrically over a wide range of capillary numbers for both smooth and well-characterized rough substrates, and for clean and surfactant interface cases. In view of the dependence of the lifetime of a film on the type of liquid and substrate, and liquid-gas and liquid-solid interfaces, we characterized the range of measurability of the film thickness in the parameter space defined by the withdrawal capillary number, the surfactant concentration, and substrate roughness size. We then study experimentally the effect of a film thickening due to the presence of surfactants. Our recent theory based on a purely hydrodynamic role of the surface active substance suggests that there is a sorption-controlled coating regime in which Marangoni effects should lead to film thinning. However, our experiments conducted in this regime demonstrate film thickening, calling into question the conventional wisdom, which is that Marangoni stresses (as accounted by the conventional interfacial boundary conditions) lead to film thickening. Next we examine the effect of well-characterized substrate roughness on the coated film thickness, which also reveals its influence on wetting-related processes and an effective boundary condition at the wall. In particular, it is found that roughness results in a significant thickening of the film relative to that on a smooth substrate and a different power of capillary number than the classical Landau-Levich law. (C) 2005 American Institute of Physics. [DOI: 10.1063/1.2112647]
\end{abstract}

\section{INTRODUCTION}

The classical result of Landau and Levich ${ }^{1}$ for the thickness of a liquid film entrained in the process of withdrawing a substrate with speed $U$ in the gravity field $g$,

$$
\bar{h}_{\infty}=0.945 l_{c} C a^{2 / 3} \quad \text { with } \quad l_{c}=\sqrt{\frac{\sigma}{\rho g}}, C a=\frac{\mu U}{\sigma},
$$

where $\rho, \mu$, and $\sigma$ are liquid density, viscosity, and surface tension, respectively, remains one of the central results in interfacial fluid mechanics. There is a large body of literature devoted to different improvements and extensions of the Landau-Levich law. ${ }^{1}$ Gravity corrections were accounted for by White and Tallmadge, ${ }^{2}$ and their incorrect approximation of the normal stress was corrected in the work of Spiers et al. ${ }^{3}$ Later, Wilson ${ }^{4}$ put ad hoc treatments by previous authors on the ground of systematic perturbation theory, establishing Eq. (1) as the leading term in an asymptotic expansion for small $\mathrm{Ca}$. This law has been also extended to the case of non-Newtonian (power law) fluids by Tallmadge ${ }^{5}$ and plastic-viscous fluids by Deryaguin and Levi, ${ }^{6}$ the effects of inertia by de Ryck and Quéré, ${ }^{7}$ and the effects of Marangoni stresses by Ramdane and Quéré. ${ }^{8}$ All these theoretical considerations, based upon macroscopic analysis, assume the existence of the film for the range of studied parameters and neglect any microscopic effects (in this paper we refer to macroscopic as defined from purely hydrodynamic considerations, while microscopic implies effects due to microscopic physics not accounted for in the standard macroscopic equations and interfacial boundary conditions). Moreover, the study of surfactant effects is far from being complete and leaves a number of questions open. Our work addresses some of these questions. Here we (1) study the existence of a film for various surfactant and substrate roughness conditions, and their effect on the measurability of film thickness, (2) provide experimental evidence that the thickening of a coating created from surfactant solutions is not due to Marangoni forces when surfactant transport is not diffusion limited, and (3) systematically evaluate the effect of substrate roughness on the film thickness. While we provide the above studies with theoretical interpretations, the main focus in this paper is on the experimental side.

Most film thickness measurements have been conducted with variations of gravimetric techniques, starting with the original experiments on wire coating by Goucher and Ward, ${ }^{9}$ and cylinder coating by Morey ${ }^{10}$ (who observed the 2/3 power law in speed with $5 \%$ accuracy before its theoretical prediction) and Deryaguin and Titievskaya. ${ }^{11}$ The latter accounts for the "lower edge effect" in finite length plates by additional experiments for a shorter immersion length $l^{\prime}$, so that actual film mass is determined on a portion of a substrate length $l-l^{\prime}$ above the lower edge, where $l$ is the original 
immersion depth [cf. also formula (2) in Sec. II]. While gravimetric measurements are still used in modern studies, such as fiber coating by Shen et al. ${ }^{12}$ and by Ramdane and Quéré, ${ }^{8}$ there are some other direct alternative techniques. For example, van Rossum ${ }^{13}$ and Kizito et al. ${ }^{14}$ used a micrometer to measure film thickness on a belt, while Spiers et $a l^{3}$ exploited capacitance probes. However, the direct measurement approaches are limited by a short lifetime of the film, since in many cases the film is destroyed almost immediately after leaving the bath. Therefore, we use a variation of gravimetry, described in Sec. II.

The possible short lifetime of the film suggests the first natural question to address from the experimental side-a measurability of film thickness (the verifiability of the Landau-Levich law being a subset of it). Quéré ${ }^{15}$ alluded to this question, referring to a transition between dry and wet regimes, but this transition is not in 1-1 correspondence with measurability [a stable film may not exist, but its (hypothetical) thickness is still measurable. For example, in fiber coating experiments, film rupture into droplets is observed and usually attributed to the Plateau-Rayleigh instability]. To our knowledge there are no systematic and self-consistent studies of the feasibility of such measurements; such a study constitutes the first objective of our work. The determination of the range of measurability is based on the extension of Derjagin's idea of the lower edge effect, which allows us to define the transition from the case when the coated liquid stays on a substrate (even though the film may rupture), and the case when part of liquid flux goes back into the bath, thus preventing measurements of the film thickness. This transition naturally depends on the characteristic time of the withdrawal process compared to that of film instabilities and rupture. The latter in turn depend on the nature of the liquid and substrate and the interaction between them. It is also generally argued that this film dewetting does not affect the film thickness measurements. ${ }^{12}$ As we will demonstrate in Secs. III $\mathrm{B}$ and IV B, for the planar case, this dewetting effect imposes certain limitations on the measurability, and its range is a function of fluid properties, roughness $r$ of the substrate, and the presence of surface active substances. In the case of surfactants, the measurability is determined by a transition curve in the $(\mathrm{Ca}, \mathrm{C})$ plane, where $C$ is the concentration. In the case of roughness, the transition curve reveals a universal behavior in the $\left(\mathrm{Ca}, r / l_{c}\right)$ plane, where $l_{c}$ is the capillary length. It is remarkable that a stable film on a vertical substrate can be obtained by two physically independent mechanisms: by introducing surfactants in the smooth coated substrate case, and by using a rough substrate in the case of a pure liquid.

Having determined the range of measurability, we proceed with our second objective-the study of surfactant effects on the film thickness - which is known to deviate substantially from the Landau-Levich law. Beginning with the famous observations by Groenveld ${ }^{16}$ for coating of a flat substrate and Bretherton ${ }^{17}$ for coating the inner walls of circular tubes, the departure from the Landau-Levich law-film thickening - is usually attributed to the presence of surface active contaminants. The experimental fact of film thickening was also confirmed in more controlled experiments by
Ramdane and Quéré ${ }^{8}$ and by Shen et al. ${ }^{12}$ The common feature of all these observations is a substantial deviation from the law for very thin films (Bretherton ${ }^{17}$ reported deviations for $C a<5 \times 10^{-3}$ ), which is usually explained by Marangoni stresses, i.e., to be due to the pure hydrodynamic nature of the surfactant effect-the Marangoni stresses pump additional mass flux into the film (cf. Quéré ${ }^{15}$ ). This idea led Groenveld $^{16}$ to the conclusion that the stagnation point, which is always formed in the process of flat substrate withdrawal, disappears. An analogous line of reasoning by Park ${ }^{18}$ has yielded an asymptotic theory which predicts film thickening. However, the nonlinear global analysis based on the purely hydrodynamic role of surfactants in the LandauLevich problem [R. Krechetnikov and G. M. Homsy (unpublished)] demonstrated that the stagnation point is still present at the interface and insoluble surfactant is simply swept away from the meniscus region, thus not influencing the film coating at all. Therefore, the flow topology neglected in the above mentioned asymptotic analyses defines the way the surfactant affects the solution of the problem. Similar to Park's work, ${ }^{18}$ approximate theories accounting for film thickening in the presence of soluble surfactant were developed in a number of works, e.g., Ramdane and Quéré ${ }^{8}$ for fiber coating, and Ratulowski and Chang ${ }^{19}$ for the Bretherton problem. The common feature of the above asymptotic studies is the assumption that the scales of the static and dynamic menisci are well-separated and are the same as in the nosurfactant case, i.e., the static meniscus, as determined from capillary statics, is scaled by capillary length $l_{c}$, and the dynamic meniscus (transition region), in which dynamic interfacial conditions come into play, is of the order of $l_{c} C a^{1 / 3}$ [see Fig. 6(a)]. This assumption, which is justified only for certain characteristic times of surfactant transport, namely the diffusion-limited case, ${ }^{19}$ simplifies the analysis substantially and justifies the above asymptotic treatments. However, the introduction of surfactant transport into the problem changes the definition of the static and dynamic menisci, and, as we will show in Sec. III C, in the case of sorption rate-limiting transport mechanism (as in our experiments), the static meniscus disappears and the dynamic meniscus scale becomes $\sim l_{c}$. This distinguishes our experiments from previous theoretical asymptotic studies and prevents the application of standard perturbation techniques that follow Landau and Levich $^{1}$ (cf. also Bretherton ${ }^{17}$ ), as these are applicable only to the case when the meniscus is static almost everywhere except for a very short transition region matching the static meniscus to a thin film region. The full nonlinear modeling of this regime [R. Krechetnikov and G. M. Homsy (unpublished)] leads to the unexpected prediction of film thinning. Thus there is no definitive combination of theory and experiment to confirm unambiguously that thickening is due to Marangoni effects. While our measurements show that the presence of surface active substances leads to thickening, which however becomes weaker for thicker films, we demonstrate with the knowledge of the kinetic properties of the surfactant that the origin of thickening in this range of parameters is not likely due to Marangoni stresses.

The second effect leading to deviations from the 
Landau-Levich law-the presence of substrate roughness-is our third objective and the subject of Sec. IV. Even though the importance of surface roughness in coating processes is well recognized, its many aspects are not understood yet. Among the known effects of roughness is a modification of heat transfer, ${ }^{20}$ stability and deposition of coatings, ${ }^{21}$ the friction coefficient in shear flows, ${ }^{22,23}$ and motion of air bubbles in capillary tubes. ${ }^{24}$ It is interesting to note that in this last work, Chen observed a substantial deviation of the film thickness at $C a \leqslant 8 \times 10^{-4}$ and attributed it to the presence of roughness. However, no quantitative characterization of either this effect or the magnitude of the roughness was made. It is natural to expect that the influence of roughness depends on the type of asperity, which ultimately is determined by the process of its production. Discussion of the sanding process used here to prepare a roughened substrate is given in the Appendix. A naturally connected problem - characterization of roughness - is thoroughly treated in Sec. IV A. Next, in Sec. IV C, we show that effects of roughness size leads to a different power in the Landau-Levich law, which can be explained by the introduction of a slip model. It is notable that roughness has a substantial influence on the stability and thickness of the film even though the rms roughness is much smaller than the film thickness. This indicates that the effects of roughness exhibit themselves in wetting-related processes as well as in a modification of the boundary condition at the wall. In Sec. IV B we provide a simple explanation of the film stabilization from thermodynamic considerations (static effect), and in Sec. IV D the origin of a partial slip condition (dynamic effect). The latter is used in a modification of the LandauLevich analysis, which explains the deviation from the classical result quite well.

\section{EXPERIMENTAL PROCEDURE}

We employ a gravimetric technique based on withdrawal of a finite plate from a bath, as shown in Fig. 1. As mentioned in Sec. I, most recent experiments are devoted to fiber coating, which is probably related to the ability to run a long fiber through the bath continuously and thus to get appreciable withdrawn mass. Most flat substrates do not allow rolling them through the bath in analogy with fibers (with the exception of experiments with belts ${ }^{13}$ ). Therefore, if one decides to work with a glass plate, for example, the first limitation is the finite length limited by the size of the tank, which in turn influences the accuracy of the balance since the higher the load the lower the accuracy. The second limitation is the lower edge effect, which introduces a systematic error, which in turn explains the "water paradox" of White and Tallmadge. ${ }^{25}$ However, both difficulties are easy to overcome. The limitation of finite length can be compensated by a series of identical withdrawals, which is equivalent to continuous coating, unless there are Stokes boundary layer effects. This also allows us to verify the repeatability of the measurements. The lower edge effect is accounted for by additional withdrawal of a partially dipped plate- this idea was utilized by Derjaguin and Titievskaya. ${ }^{11}$ Here we extend this idea to study the question of verifiability of the Landau-

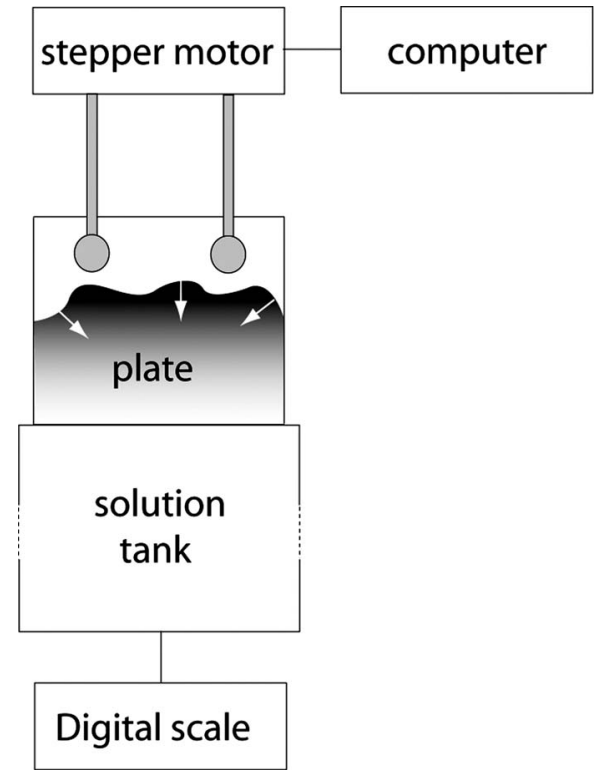

FIG. 1. Sketch of the experimental setup.

Levich law by investigating the amount of liquid going back to the bath in the course of withdrawal by measuring the film thickness as a function of varying the lower edge depth.

A schematic of the experimental setup is shown in Fig. 1. The flat substrate [glass plate(s)] is withdrawn by a computer-controlled high-precision stepper motor (Velmex, Inc.) from a tank of inner dimensions $20 \times 110 \times 120$ (depth, width, height in $\mathrm{mm}$ ). The tank is fixed on the pan of a digital balance Explorer Pro EP 612C (Ohaus, Inc.) with readability $0.01 \mathrm{~g}$. The setup was protected with a windshield to minimize the effect of the ambient environment.

Assuming that the number of withdrawals and change of mass for measuring film thickness on the plate of width $w$ with dipping depth $l$ are $N$ and $\delta m$, and the lower edge effect with depth $l^{\prime}$ was $N^{\prime}$ and $\delta m^{\prime}$, the film thickness is determined from

$$
h=\left(\frac{\delta m}{N}-\frac{\delta m^{\prime}}{N^{\prime}}\right) \frac{1}{2\left(l-l^{\prime}\right) w \rho},
$$

where the factor 2 comes from two sides of the plate. The mass deposited on the lateral edges was also taken into account in the combination of the study of its wettability (wetting properties of the glass are different on the main and cut surfaces); the error induced by possible inaccuracies with these side edge mass deposition is less than $1 \%$ in view of the high aspect ratio of substrate width to thickness $(\sim 50)$.

The experiments are performed with water-based surfactant solutions of various concentrations, the viscosities of which are controlled by adding glycerol. In view of the nature of these liquids and an ambient temperature $22-25{ }^{\circ} \mathrm{C}$, evaporation effects can be neglected, as was verified experimentally. In view of the temperature dependence of fluid parameters (surface tension, viscosity, and density), the temperature was recorded for each set of measurements, and the corresponding adjustments has been made with the use of tables of physical properties. ${ }^{26-28}$ The properties of the surfactant used are reviewed in Sec. III A. For repeatable mea- 
surements, the water was de-ionized (DI) and freshly prepared. While the former allows us to work with the same type of water, the latter is crucial since DI water is aggressive and adsorbs ions from environment. Glycerol and surfactant solutions were prepared by thorough mixing with a Pyro-Magnestir (Lab-Line Instruments, Inc.).

\section{DIP COATING IN SURFACTANT SOLUTIONS}

We start the discussion with the influence of surfactant. First, in Sec. III A, we review the properties of the surfactant used in our experiments since some of its physical characteristics are crucial in the interpretation of the results. Next, in Sec. III B, we continue the discussion of the lower edge effect mentioned in the introduction and Sec. II, with the help of which we determine the range of measurability of film thickness. In the same subsection we discuss the physical reasons for the limits of measurability as coming from the film instability and dewetting processes.

\section{A. Physical properties of SDS}

We used a well-characterized surfactant-sodium dodecyl sulfate (SDS). In this subsection we recall the basic equilibrium and nonequilibrium kinetic and material properties of SDS which are necessary for further discussion. A more detailed description is given in Fernandez et al. ${ }^{29}$ To denote the SDS concentration we will be using either upper case $C$, which is always dimensional by the context, or lower case $c$, which is nondimensional and defined as $c=C / C_{m}$ with $C_{m}$ being the critical micelle concentration (CMC) of $8.3 \mathrm{mM}$.

SDS (chemical formula $\mathrm{C}_{12} \mathrm{H}_{25} \mathrm{NaO}_{4} \mathrm{~S}$ ) is ionic with a molar mass of $288.380 \mathrm{~g} / \mathrm{mol}$. The analysis in Ref. 30 of the data by Ref. 31 gives the value for the Langmuir constant

$$
K_{L}=\frac{k_{a}}{k_{d} \Gamma_{m}}=0.11 \frac{\mathrm{m}^{3}}{\mathrm{~mol}},
$$

which is in a good agreement with equilibrium adsorption data. The material behavior of SDS, $\sigma(C)$, is given in Ref. 32 , measured by the drop volume method.

While in the nonequilibrium case there is a dependence of the kinetic constants on concentration, ${ }^{33}$ one can approximate the nonequilibrium surfactant dynamics with the Langmuir-Hinshelwood equation, valid below CMC, for the surfactant surface concentration $\Gamma$ :

$$
\frac{d \Gamma}{d t}=k_{a} C\left(1-\frac{\Gamma}{\Gamma_{m}}\right)-k_{d} \Gamma
$$

with $k_{a}=0.6410^{-5} \mathrm{~m} \mathrm{~s}^{-1}, k_{d}=5.87 \mathrm{~s}^{-1}$. Therefore, the typical time of adsorption $t_{a}=\Gamma_{m} /\left(k_{a} C\right)$ is $<1 \mathrm{~s}$, for the working range of concentrations $C \in(1.5,8.2) \mathrm{mM}$, and the time of desorption $t_{d}=k_{d}^{-1}<0.2 \mathrm{~s}$.

As one can conclude from Eq. (4), the rate at which the surfactant is supplied by adsorption to the interface, created with a speed $U$, is defined by the value of the parameter:

$$
\text { St } \kappa=\frac{t_{v}}{t_{a}}, \quad \text { where } \quad \mathrm{St}=\frac{k_{a}}{U}, \quad \kappa=\frac{l_{c} C}{\Gamma_{m}},
$$

where $t_{v}=l_{c} / U$ is a flow time (interface stretching). The use of the capillary length $l_{c}$ as the characteristic length is justified by the fact that new interface is created in the meniscus region whose characteristic scale is $l_{c}$ and the transport of surfactant between bulk and interface happens primarily in this region as will be shown in Sec. III C. Therefore, if the parameter St $\kappa$ is greater than unity, the surfactant is rapidly supplied to the interface, thus compensating for the dilution created by interface stretching and not allowing Marangoni stresses to be produced. This fact suggests a simple test to understand the origin of film thickening in the presence of surfactants. If for large values of this parameter thickening is still present, the effect is definitely not due to Marangoni stresses, but to some other physical phenomena. Further discussion is given in Sec. III C.

\section{B. Measurability region and film stability}

The experiments started with verifying the absence of nonintentional (noncontrollable) contaminants. The film thickness measured on a glass plate withdrawn from freshly de-ionized water shows a maximum in the first one or two dip coatings, and then attenuates to a constant value insensitive to the time of immersion. The last fact insures the validity of measurements and absence of contamination from the environment. A large number of experiments (not shown) in the case of clean interface using water-glycerol solutions over a wide range of $C a, 10^{-3} \leqslant C a \leqslant 10^{-1}$, showed agreement with Eq. (1), with small corrections for the finite bath size and for finite $\mathrm{Ca}$ and an overall accuracy of $10 \%$. In particular, the $2 / 3$ power law was verified with an accuracy of $5 \%$.

As a first step we studied the functional dependence of film thickness on the lower edge depth, as described in Sec. I and by Eq. (2). Ideally, the true lower edge effect should result in a film thickness slightly higher than that computed with the lower edge effect subtracted, and the resulting picture should be similar to the dashed line for $C a=5.1 \times 10^{-4}$ in Fig. 2 [in which the lower edge depth is defined as a percentage of the whole length, $\left.\left(l-L^{\prime}\right) / l\right]$. It is known that in the course of withdrawal a very thin film has a short life since it is destroyed by film instability, which eventually leads to rupturing and dewetting. This mechanism may lead to a decrease in the measured mass, and thus the inferred film thickness. Referring again to Fig. 2, obtained for dip coating of a glass plate roughened with 400-grit sandpaper in $20 \%$ glycerol-DI water mixture, the film thickness for capillary numbers $5.1 \times 10^{-4}$ and $9.2 \times 10^{-4}$ obeys the ideal behavior in which the inferred film thickness is independent of the lower edge depth. However, below $C a=5.1 \times 10^{-4}$, e.g., for $\mathrm{Ca}=1.6 \times 10^{-4}$, the film thickness is substantially influenced by the dewetting process-part of the mass flux goes back into the tank. Accordingly, the capillary number $\mathrm{Ca}=5.1$ $\times 10^{-4}$ designates the transition between regimes of measurability and nonmeasurability.

Performing this analysis for each concentration $C$ and 


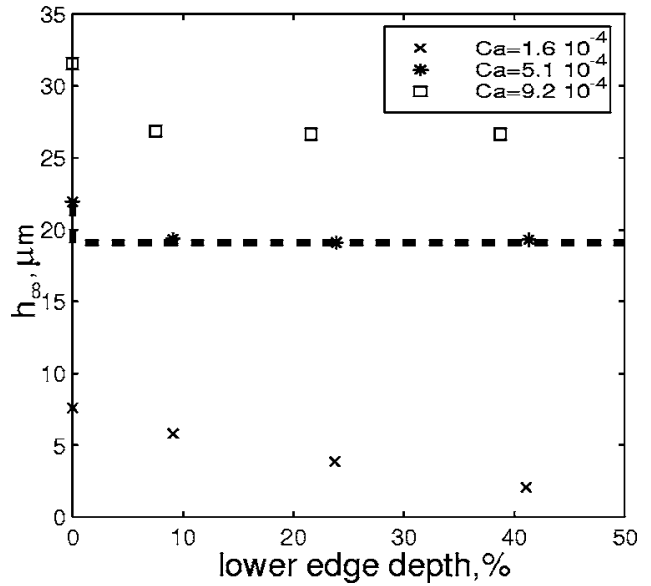

FIG. 2. Lower edge effect. The dashed line represents a transition from measurability to nonmeasurability of the film thickness and the magnitude of $h_{\infty}$ corresponding to it is lower than that without the lower edge effect, as expected.

speed of withdrawal, we obtain the region of measurability as a function of the speed of withdrawal and concentration, which is shown in Fig. 3. The dimensional and scaled versions, Figs. 3(a) and 3(b), respectively, both exhibit mono-
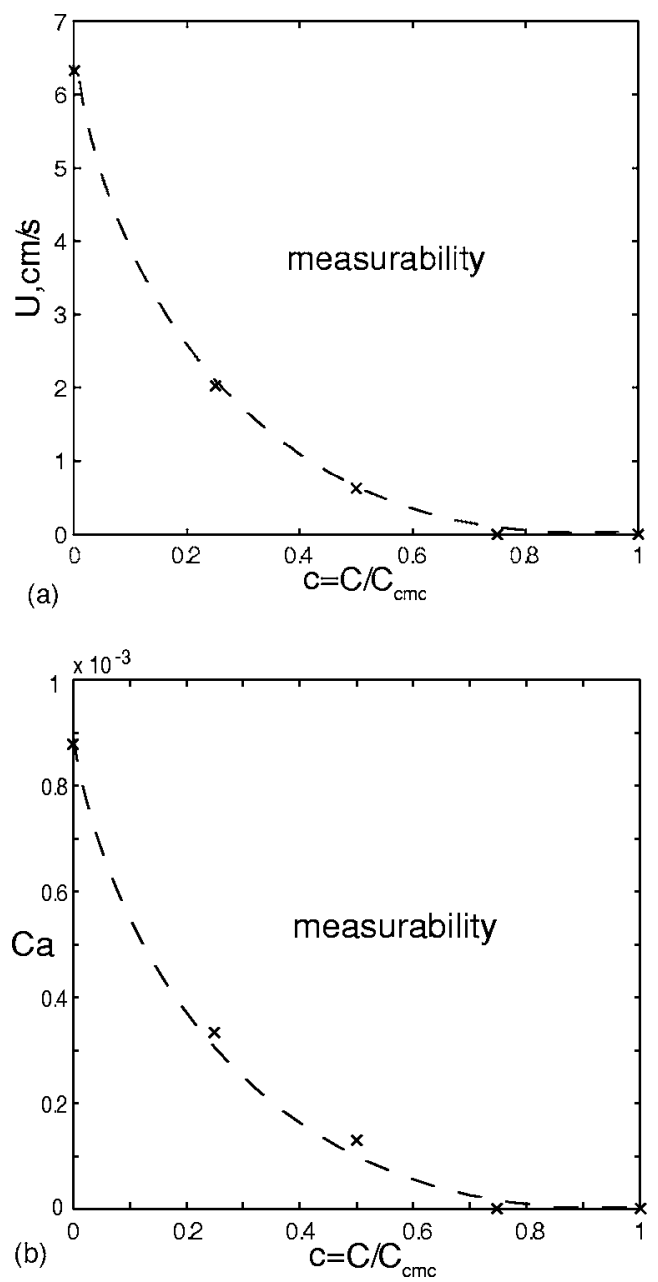

FIG. 3. On measurability of film thickness for a water-surfactant solution on a smooth substrate. Transition curve: (a) dimensional, (b) scaled. tonic dependence [even though the capillary number in Fig. 3(b) has a dependence on the concentration through the variation of surface tension]. It is also notable that as the concentration approaches CMC the measurability becomes feasible for any withdrawal speed $U$ above zero. This is the result of the film stabilization by surfactants.

As demonstrated by these experiments, the finiteness of the measurability region comes from film rupture and dewetting, the physical reasons of which represent an active area of current study, primarily for horizontal substrates (no influence of gravity). While the question of the stability of a thin film on a horizontal substrate is still a subject of ongoing research (e.g., Refs. 34 and 35), the film stability on a vertical substrate is even more complicated and has a very rich history. Disturbances may originate from gravity drainage (which is characterized by the maximum speed $\sim B o_{h}^{1 / 2} \sigma / \mu$, which is $\sim 10^{-5} \mathrm{~cm} \mathrm{~s}^{-1}$ in our case), from thermal fluctuations of a liquid surface, ${ }^{36}$ from the inhomogeneous surface energy of the substrate (fluid moves from the less wettable to the more wettable regions), etc. While their linear amplification (cf. Benjamin ${ }^{37}$ and Yih $^{38}$ ) explains instability and accounts for the thinning stage, the nonlinear regime is responsible for the eventual breakup (rupture) and is usually attributed either to growing fluctuation waves (an idea due to Scheludko) or to nucleation inside the film (an idea due to Derjaguin). Though both mechanisms have been detected, ${ }^{39}$ the first is the most studied one. Its evolution has been modeled with London-van der Waals attraction and double-layer repulsion by Vrij, ${ }^{40}$ the London-van der Waals force by Williams and Davis, ${ }^{41}$ short- and long-range forces by Sharma $e t$ $a l .{ }^{34,42,43}$ and the effects of drying by Schwartz et al. ${ }^{35}$ For pure liquids it is believed that film rupture is due to competition of long- (apolar, van der Waals) and short-range polar forces, resulting in an intermolecular interaction free energy of the form $\Delta \mathcal{F}=-A / 12 \pi h^{2}+S \exp (-h / l)$. In general, the stability characteristics depend upon the orientation of double layers at both interfaces, which in turn are influenced by the type of solvent and surfactant molecules. The above discussion provides a qualitative picture behind the film instability rupture and dewetting observed in our experiments.

Concluding, we mention that our measurability results provide an interpretation for the classical experimental observations which indicate a strong dependence of the film thickness on the following:

(1) The charge on the substrate. Introduction of electrostatic charge on a clean glass rod by rubbing with rabbit fur ${ }^{44}$ also led to substantial deviations from theoretical predictions of Landau-Levich: the higher the charge the lower the thickness.

(2) The cleaning procedure. Acid cleaning of the substrate versus an acetone rinse resulted in $+35 \%$ deviation from Landau-Levich law versus $-15 \%$, respectively. ${ }^{44}$

In our opinion, these particular deviations are due to working in a regime when film thickness is not measurable and thus a small change in wettability leads to substantial variation of the entrained mass. 


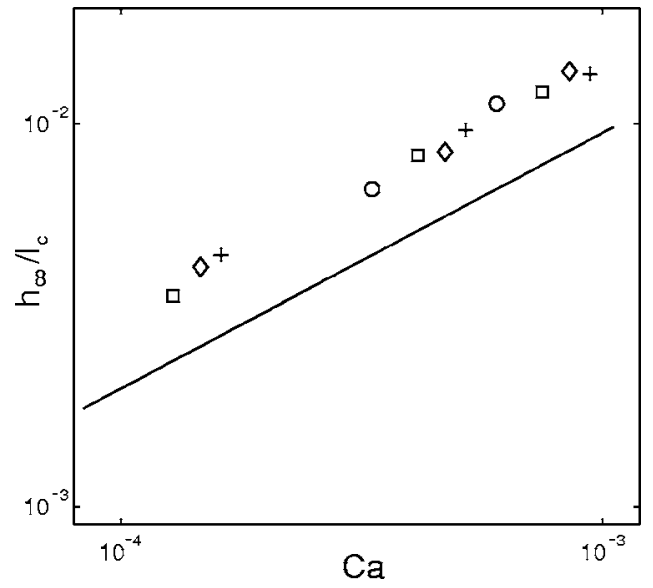

FIG. 4. Data for coating of surfactant solution. SDS concentration, as fraction of CMC: $\bigcirc, 0.25 ; \square, 0.50 ; \diamond, 0.75 ;+, 1.00$. The solid curve corresponds to theory (1) with surface tension evaluated at the corresponding concentration and taken as constant.

\section{Film thickening}

Having established the accuracy of our technique and defined the range of measurability, we proceed with the study of the film thickness in the presence of surfactant. Figure 4 shows typical data for dip coating from a surfactant solution. In order to separate the surfactant dynamical effects from simple lowering the surface tension, it was suggested ${ }^{12,15}$ to use the so-called thickening factor, the ratio of the measured film thickness to the theoretical one for a uniformly distributed surfactant (no concentration gradients)

$$
\alpha=\bar{h}_{\infty} / \bar{h}_{\infty}^{\text {theory }},
$$

where $\bar{h}_{\infty}^{\text {theory }}$ is given by formula (1). Our results for $\alpha$ are shown in Fig. 5 for water-surfactant solutions. In view of the limited range of withdrawal speeds, the range of measurability in Fig. 3 makes measurements legitimate only for $C / C_{c m c}>0.2$. In view of repeatability of the points in Fig. 5 and small errors relative to variations around the average value of the thickening factor $\alpha=1.55$, one can observe a nonmonotonic dependence of $\alpha$ on the speed of withdrawal

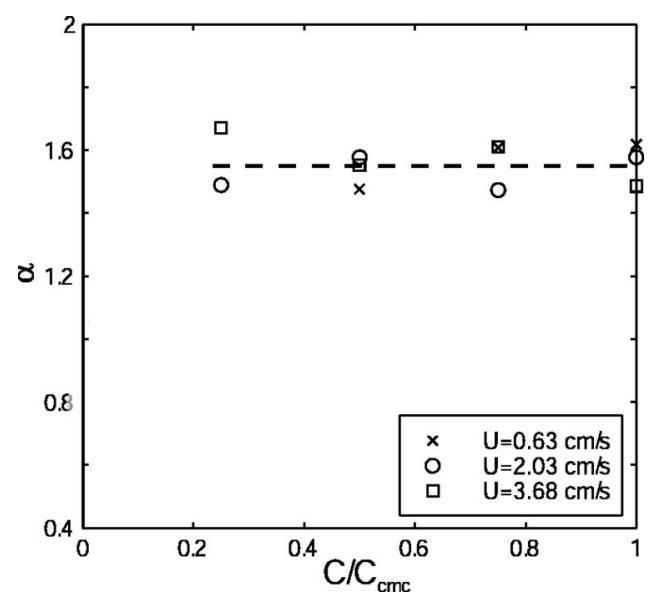

FIG. 5. Thickening factor $\alpha$ (the size of symbols represent the error bar; the dashed line represents an averaged value of $\alpha$ ).

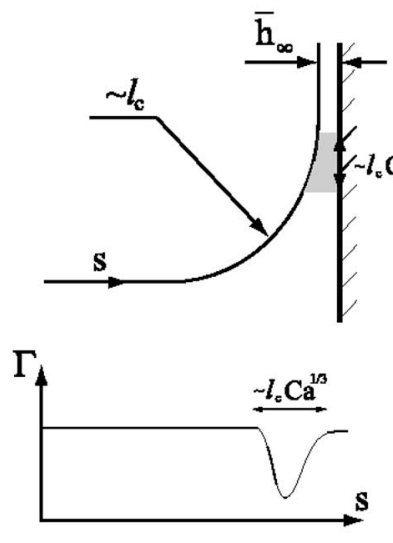

(a)
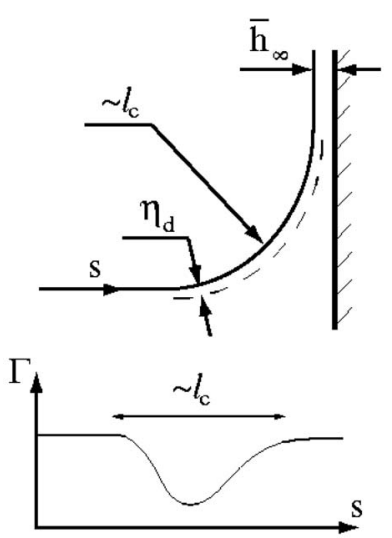

(b)
FIG. 6. On the definition of dynamic and static menisci. $s$ is the arc length. (a) Standard asymptotic analysis (Refs. 18 and 19). (b) Fully dynamic meniscus.

$U$ and concentration $c$, which indeed could be due to Marangoni effects, since the concentration gradients depend not only on the rate of interface stretching but also on the kinetic constants. The nonmonotonicity indicates the complicated physics of the phenomena and to the authors belief is not due to any experimental inaccuracy. It is also interesting to note the absence of a well-defined maximum of $\alpha$, in contrast with fiber coating experiments. ${ }^{15}$

In order to understand if the presence of surfactant leads to Marangoni stresses generating film thickening, we first review the assumptions on which existing asymptotic analyses of the effect of surfactants are based. ${ }^{18,19,45}$ Figure 6(a) shows a schematic of that situation: in the case of a clean interface, the static meniscus with characteristic length $l_{c}$ is joined to an entrained film of uniform thickness through a transition layer in which viscous drag and capillary pressure gradients balance. The spatial extent of this layer is well known to be $O\left(l_{c} \mathrm{Ca}^{1 / 3}\right)$ and the film profile is governed by the Landau-Levich equation. ${ }^{1,17}$ The conventional asymptotic analyses of surfactant effects ${ }^{18,19,45}$ make a key assumption - surfactant gradients are limited to the transition layer defined for the clean interface problem - which leads to the bottom schematic in Fig. 6(a), i.e., surfactant gradients are limited in spatial extent, thus allowing for a local analysis. Also, in order to justify this assumption of locality, it is necessary that no stagnation points occur within the transition layer, as explicitly discussed in the case of insoluble surfactant by Park. ${ }^{18}$ In the case of a soluble surfactant there are two transport models within the limits of the above key assumption: sorption-limited transport, which leads to film thinning as predicted by Ginley and Radke, ${ }^{45}$ and masstransfer-limited transport, which accounts for film thickening due to bulk concentration gradients in the work of Ratulowski and Chang. ${ }^{19}$ All these assumptions and restrictions together establish a scale for Marangoni stresses appearing in the tangential stress balance that limit all existing analyses to dilute surfactant concentrations. This in turn allows the effects to be computed locally within the transition layer and within the approximations of lubrication theory. The main intrigue of our experimental study is that even though the 
surfactant transport is sorption limited, the film is still thickened (as opposed to the study by Ginley and Radke ${ }^{45}$ ). The origin of this behavior is in the global influence of the surfactant dynamics as shown in Fig. 6(b) and will be discussed below. Therefore none of the above local theories is applicable in this case.

Our experiments are conducted in a range of parameters for which none of the lubrication theories assumptions hold, which may be established by order of magnitude estimates based on the kinetic properties of SDS discussed in Sec. III A. First, we demonstrate that for the conditions of our experiments, the meniscus is dynamic everywhere, i.e., its scale is $\sim l_{c}$. Indeed, the fact that the characteristic time of surfactant adsorption $t_{a}$ and of interface stretching $t_{v} \sim l_{c} / U$ are of the same order of $0.2 \mathrm{~s}$ for $U \sim 1 \mathrm{~cm} \mathrm{~s}^{-1}$ leads to the inevitable conclusion that the concentration $\Gamma$ changes in the leading order, $\delta \Gamma \sim \Gamma$, over the whole meniscus scale $l_{c}$ as shown in Fig. 6(b). This is also confirmed by full numerical modeling [R. Krechetnikov and G. M. Homsy (unpublished)]. Next, we demonstrate that the transport in our case is sorption limited. Since the interface stretching takes place along the whole meniscus of characteristic length $l_{c}$, the number of molecules necessary to fill the interface per unit length in the third dimension is $\Gamma \cdot \eta_{d}$ and will be consumed from the sublayer of thickness $\eta_{d}$ near the interface [cf. Fig. 6(b)], $C l_{c} \eta_{d}=\Gamma l_{c}$. Accordingly, a sublayer thickness is $\eta_{d}$ $\sim \Gamma / C$. The corresponding diffusion time is $t_{\text {diff }} \sim \eta_{d}^{2} / D$, where $D=8 \times 10^{-10} \mathrm{~m}^{2} / \mathrm{s}$ is a typical value for the diffusion coefficient. As a result, for SDS at $C=C_{c m c}=8.2 \mathrm{~mol} / \mathrm{m}^{3}$, for example, we have $\Gamma=\Gamma_{m}=10^{-5} \mathrm{~mol} / \mathrm{m}^{2}$, so that $\eta_{d} \sim 1 \mu \mathrm{m}$, and diffusion time $t_{\text {diff }} \sim 10^{-3} \mathrm{~s}$, which is negligible compared to the adsorption time $t_{a} \sim \Gamma_{m} / k_{a} C \sim 0.2 \mathrm{~s}$. Thus, the transport is sorption limited. Notably, the value of $\eta_{d}$ $=1 \mu \mathrm{m}$ also implies that the bulk concentration can be treated as constant, since surfactant adsorption and transport does not change the bulk concentration in any significant way. This fact is, of course, determined by the thin film region, where one would expect the change to be largest, but $\eta_{d} / h_{\infty} \ll 1$ in this range of parameters. This regime is different from the case considered by Ratulowski and Chang, ${ }^{19}$ who investigated the limit of trace amounts of surfactant, $\eta_{d} \sim h_{\infty}$.

Based on the above conclusions of dynamic meniscus scale $\sim l_{c}$ and sorption rate-limiting transport, we can make the following observations, which indicate that the observed film thickening is not likely to be due to Marangoni stresses. First of all, Eq. (4) indicates that the adsorption becomes faster for higher concentrations. Therefore, for a given speed of interface stretching, which is related to the speed of withdrawal, Marangoni stresses due to concentration gradients become negligible if the product St $\boldsymbol{\kappa}$ is above unity. In this context, Fig. 7 showing $h_{\infty} / l_{c}$ vs St $\cdot \kappa$ convincingly demonstrates the unimportance of Marangoni effects. The same thickening behavior persists to very large values of $\mathrm{St} \cdot \boldsymbol{\kappa}$ (not shown), accomplished by doing experiments at $C / C_{c m c}$ $=10{ }^{46}$ Another indication of the negligible effect of Marangoni stresses follows from our modeling [R. Krechetnikov and G. M. Homsy (unpublished)], based on a purely hydrodynamic effect of surfactant, which demonstrated that

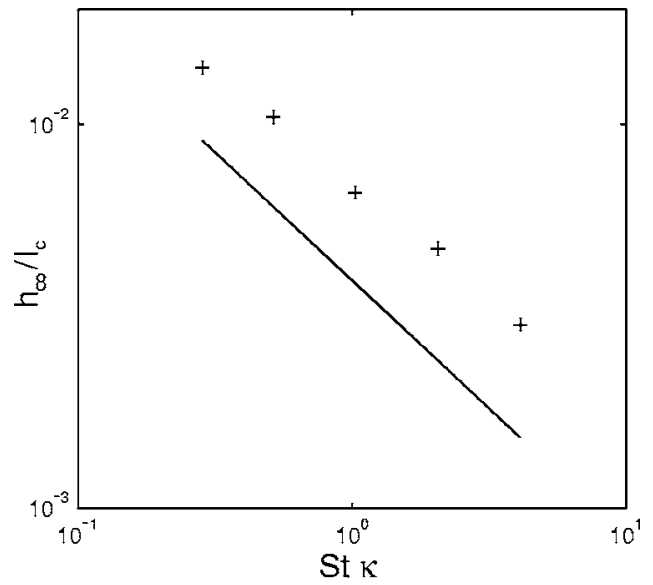

FIG. 7. Film thickening in the case of fast adsorption. The solid curve corresponds to theory (Ref. 1).

in this case the Marangoni stresses would depend on the capillary number and thus substantially distort the $2 / 3$ power law, or putting it another way, they would lead to a dependence of $\alpha$ on $C a$. However, as seen from Fig. 4, the effect of film thickening is $\mathrm{Ca}$ independent, and thus in this regime of relative kinetic/hydrodynamic/diffusion time scales, the effect cannot be explained by any of the existing analyses of Marangoni stresses. It should be noted that this regime is different from that considered by Quéré, ${ }^{15}$ who made the standard assumptions that the Marangoni stresses are restricted to a short region of length $l_{c} \mathrm{Ca}^{1 / 3}$ and the variation of the surfactant concentration is small, $\delta \Gamma \sim \Gamma C a^{2 / 3}$, as shown in Fig. 6(a). In contrast, in our case the Marangoni stresses work on the whole meniscus $\sim l_{c}$ and are of the order of $1, \delta \Gamma \sim \Gamma$. The latter is allowed by the existence of an interfacial sublayer of thickness $y \sim \tilde{y} C a^{1 / 3}, \tilde{y} \sim O(1)$, discussed in Ref. 47. Within this sublayer the viscous and Marangoni stresses are equilibrated according to

$$
\frac{\mathrm{d} \sigma}{\mathrm{d} \Gamma} \frac{\partial \Gamma}{\partial x}=C a^{2 / 3} \frac{\partial u}{\partial y}=C a^{2 / 3} \frac{\partial^{2} \psi}{\partial y^{2}},
$$

where $x$ is a coordinate along the interface and $y$ is a normal coordinate. All previous asymptotic studies exploited only one possibility, $\delta \Gamma \sim C a^{2 / 3} \Gamma$, to achieve balance between viscous and Marangoni stresses.

As mentioned in Sec. I, it is generally argued that the thickening observed in the presence of surfactants is due to Marangoni stresses originating from surfactant concentration gradients. However, as numerical modeling of the ideal Landau-Levich problem based on a purely hydrodynamical role of surface active substance demonstrated [R. Krechetnikov and G. M. Homsy (unpublished)], the effect is the opposite one (thinning), and therefore the thickening observed experimentally is likely due to some other mechanism coming into play when surfactants are present. The above conclusion should stimulate further research in this direction; while we do not offer any explanation at this stage, we offer the following comments on a number of possibilities one might think of and reasons to discard them. 
In view of the film thickness being in the microscopic range, $\bar{h}_{\infty} \sim 1-20 \mu \mathrm{m}$, there are several possible effects to consider. The first one is somewhat connected with the next section on dip coating of rough substrates-it is known that adsorbed surfactant molecules can appear to act as a somewhat rougher surface, ${ }^{48}$ thus changing the boundary condition from "stick" to "partial slip." However, we have to discard this possibility in view of the fact that SDS molecules are small and that formation of micelles takes place only above CMC. The second possible effect may come from changes of rheological properties of a thin film compared to those of a bulk. It is known that the presence of SDS in quantities used in our experiments $(<1)$ and CMC does not lead to noticeable change in viscosity, but only in surface tension. ${ }^{12}$ However, it is expected that the properties of liquid (even pure) in a thin film are affected by the interaction with molecules of the solid, the presence of double layers at the interfaces (electroviscous effect-resistance to flow of the counterions in the diffuse double layers), charged surfaces (viscoelectric effect), and so on. Measurements by Israelachvili ${ }^{49}$ demonstrated that the viscosity of water is within $10 \%$ of its bulk value in films as thin as $50 \AA$. As demonstrated by so-called "blow-off"experiments, ${ }^{50}$ viscosity is a structure-sensitive property for films of polar liquids and of thickness up to $10^{-5} \mathrm{~cm}$ (both conditions should be met simultaneously). Apparently, this is not enough to explain our results. Another structural effect could be in exhibiting viscoelastic properties of thin surfactant films as found by Kutzner et al. ${ }^{51}$ However, it is not clear for which film thickness range this effect is substantial-it should depend on the nature of the surfactant as well. The third possibility in this list is the disjoining pressure-the interaction of the liquid (or solution) and substrate through London-van der Waals forces, diffuse double layers, and structural forces. It is known that electrical double-layer forces in the silica/ water system dominate over the London-van der Waals forces for film thicknesses greater $300 \AA$. This fact was verified experimentally ${ }^{52,53}$ by film thickness measurements in the presence of salt(s), which is known to suppress the double-layer interaction; the film thickness decreases with an increase of salt concentration and with an increase of valency of the cation. The nature of the double layer at the silica/ water interface is due to the dissociation of terminal silanol groups, the degree of which depends on the water type (in view of the aggressive nature of de-ionized water, the dissociation is the strongest): silica acquires a negative charge of $0.32 \mathrm{mC} / \mathrm{m}^{2}$ with a double-layer thickness $l_{D}$ $=0.275 \mu \mathrm{m} .{ }^{54,55}$ Rough or porous silica surfaces lead to much higher charge. ${ }^{56}$ It is obvious that these electrical in origin forces should reveal themselves not only in the thickness of a coated film but also in its stability characteristics. Indeed, their strong effect on the film stability was found experimentally at very great thicknesses, $10^{-2} \mathrm{~cm}$, by the experiments of Padday ${ }^{57}$ on film rupture and theoretically confirmed by Mitlin, ${ }^{58}$ who described the dewetting theory in analogy with the Cahn theory of spinodal decomposition. The effect of the inclusion of aqueous salt and surfactants ${ }^{57}$

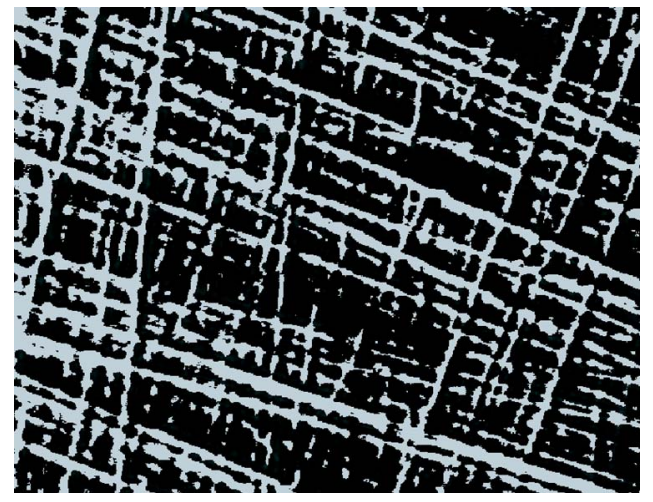

FIG. 8. Interferometric image $(230 \mu \mathrm{m} \times 300 \mu \mathrm{m})$ of a glass substrate roughened with 1500 -grit sandpaper.

suggests that the responsible long-range forces are electrical in origin. However, the dynamic effect on thickness of a coating film has not been studied.

Concluding, one has to admit that none of the possibilities above is capable of explaining the observed film thickening at the level of current knowledge, as the pure hydrodynamic effect of the surfactant-Marangoni stresses-is not capable as well.

\section{DIP COATING OF ROUGH SURFACES}

In this section we study the dependence of film thickness on surface roughness. The outline of this section is as follows: we first perform a characterization of roughened substrates in Sec. IV A, with additional information on the roughness production given in the Appendix. We then follow a program similar to that of Sec. III of our surfactant study, i.e., in Sec. IV B, we examine the range of measurability and link it with the stability properties of the film and offer a simple thermodynamic (static) way to understand the stabilizing effect of roughness. In Sec. IV C we focus on dynamic effects of roughness that lead to a deviation from the law (1) and explain it on the basis of a modified boundary condition at the wall, i.e., by replacing no-slip by partial slip condition. Incorporation of the idea into the Landau-Levich analysis yields a qualitative understanding of the deviation.

\section{A. Characterization of rough substrates}

An example of a roughened substrate- sanded glassused in our experiments is shown in Fig. 8. While the sanding process represents a separate interesting field for study and is discussed in the Appendix, for our present purposes we present here just a statistical characterization of the roughness produced.

The rough substrate can be exhaustively described by a function $z(\mathbf{r})$, which specifies the "height" of the surface at the in-plane coordinates $\mathbf{r}=(x, y)$ in an appropriate reference frame. The density of the measured points $z_{i}$, obtained for example from a stylus or an interferometric profilometer, should be, of course, high enough to resolve the characteristic roughness scales. This can be checked by testing the convergence with increasing resolution of the correlation length $\xi_{\perp}$ normal to the surface, which is also known as a root- 


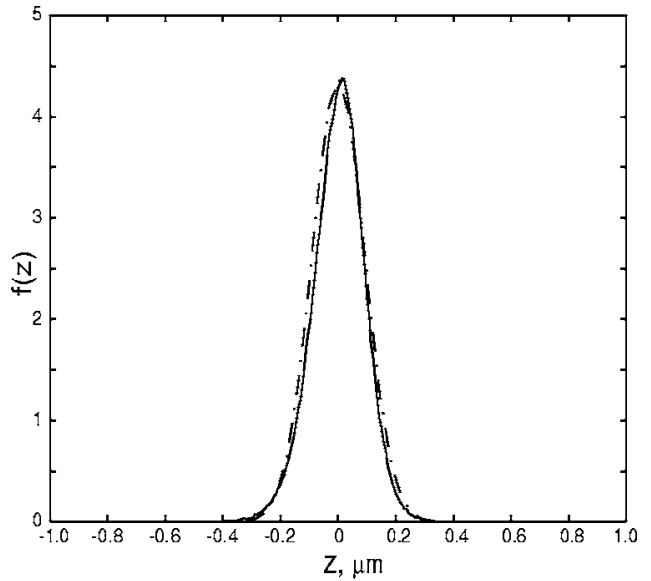

FIG. 9. Probability distribution function of roughness for a glass substrate roughened with 1500-grit sandpaper. The solid curve corresponds to the experimental pdf based on $\sim 2 \times 10^{5}$ observations. The dashed curve is a theoretical normal distribution based on estimated variance, $\sigma=91.1 \mathrm{~nm}$, and mean, $\bar{z}=0.53 \mathrm{~nm}$.

mean-square fluctuation $\sigma$ around the averaged position $\bar{z}$ $=(1 / n) \sum_{i=1}^{n} z_{i} \equiv\langle\mathbf{z}\rangle$ and equal to the square root of the variance $\sigma^{2}=\left\langle(\mathbf{z}-\bar{z})^{2}\right\rangle$. Knowledge of $z(\mathbf{r})$ also allows the definition of an average roughness,

$$
R_{a}=\frac{1}{n} \sum_{i=1}^{n}\left|z_{i}-\bar{z}\right|,
$$

denoted below simply by $r$, which will be the primary parameter for roughness characterization. Besides the average roughness $r$, the mean height $\bar{z}$ and root-mean-square fluctuation $\sigma$, the rough surface is characterized by the correlation length $\xi_{\|}$parallel to the surface,

$$
\xi_{\|}=\int \mathbf{r} \Gamma_{z} \mathrm{~d} \mathbf{r} / \int \Gamma_{z} \mathrm{~d} \mathbf{r},
$$

where $\Gamma_{z}(\mathbf{r})=\left\langle z\left(\mathbf{r}^{\prime}\right) z\left(\mathbf{r}^{\prime}+\mathbf{r}\right)\right\rangle-\bar{z}^{2}$ is the height correlation function (refer to Ref. 59) as it relates the heights at two positions $\mathbf{r}^{\prime}$ and $\mathbf{r}^{\prime}+\mathbf{r}$ and goes to zero when the heights become uncorrelated at the distance $\sim \xi_{\|}$.

Based on the above introduced mean and variance one can consider a normal (Gaussian) probability distribution function,

$$
f(z)=\frac{1}{\sigma \sqrt{2 \pi}} e^{-(z-\bar{z})^{2} / 2 \sigma^{2}} .
$$

The classical way to get the above statistics is based on stylus techniques developed in the 1930s, the basic idea of which is to record vertical deflection of a stylus when its tip is moved across a surface. Nowadays, optical profiling based on the analysis of interferometric pictures allows a more convenient way of obtaining surface coordinates. In our study we used an interferometer manufactured by Veeco Instruments, Inc.

Figure 9 shows data on roughness and a fit to Eq. (8). The small right skewness equal to 0.0932 indicates that the roughness distribution is nearly symmetric, while a kurtosis of 15.1075 (so that the distribution must be leptokurtic, that

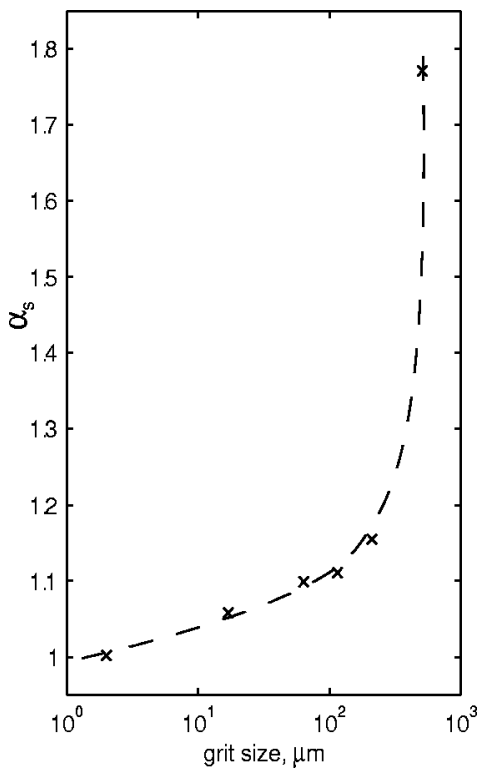

FIG. 10. Surface area index.

is, more peaked than the normal one) suggests that the data are not normal. However, Fig. 9 suggests that the distribution is not strongly peaked relative to the normal one and that the high kurtosis value might also be due to the chopping of the spectrum produced by the interferometer. Taking the natural logarithm of the data, suggested by the positive skewness, reduces the kurtosis to 5.0283 , but leads to moderate negative skewness of -0.5585 . Therefore, one can describe the measured pdf as more normal than log-normal. ${ }^{60}$ Lastly, it should be noted that sometimes it is very difficult to discern a difference between the normal and log-normal distributions, like in the case of reaction time experiment data, ${ }^{61}$ or distribution of heights of women. ${ }^{62}$ It is interesting to note that the Shapiro-Wilk $W$-statistic test ${ }^{63}$ of a small subset of $\mathbf{z}$, which is one of the best omnibus tests for normality, rejects the null hypothesis of normality at the 0.05 level of significance (i.e., we can reject the idea that $\mathbf{z}$ comes from a normal distribution with $95 \%$ confidence) giving a $p$ value of 0.02 (while the $W$ statistic is 0.984 ) and lower for all the substrates studied, including glass and metallic ones. The same result is suggested by the Lilliefors test, which is probably explained by a cutoff of the tails in the course of the interferometric measurements.

Another important surface characteristic is a surface area index - the ratio of actual to apparent areas. Its dependence on the grit size of the sandpaper used for roughening is shown in Fig. 10. This parameter is important in the analysis of the film stability in the next subsection.

\section{B. Stabilization effect of roughness: Static effect}

The study analogous to Sec. III B of the transition from coating to dewetting yields the data shown in dimensional variables in Fig. 11(a) for two liquids of different viscosities. The same transition curves in nondimensional variables, with speeds scaled by $\sigma / \mu$ and roughness scaled by the capillary length, are in Fig. 11(b). The data collapse onto one curve thus demonstrating a universal behavior. While this univer- 


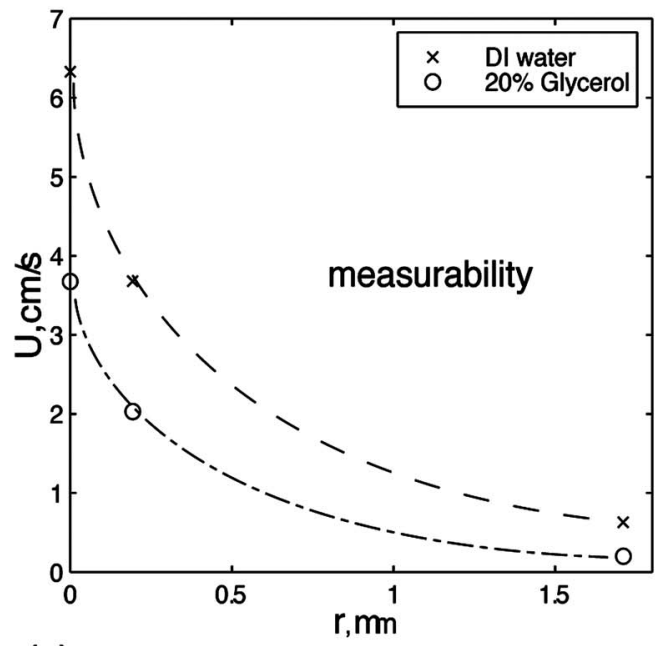

(a)

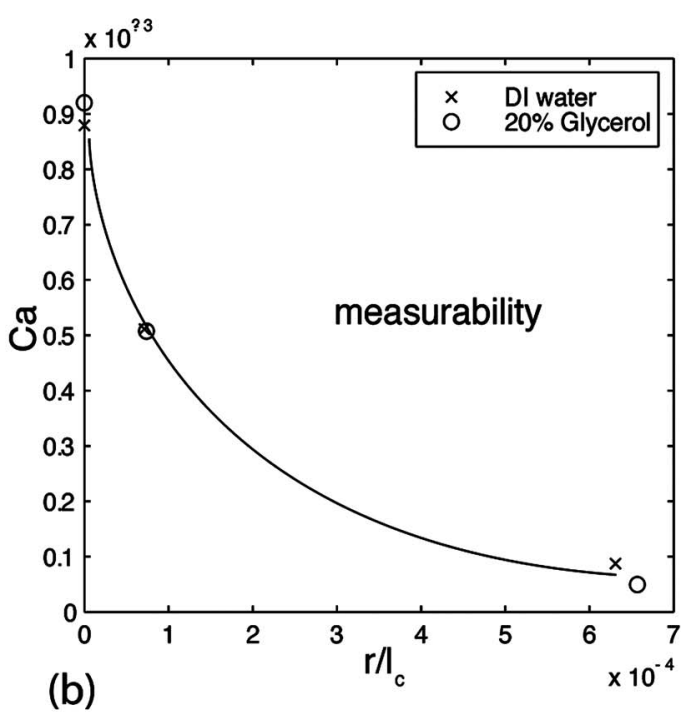

FIG. 11. On the measurability and verifiability of the Landau-Levich law. Transition curve (a) in dimensional space; $r$ stands for the average roughness (6). (b) in scaled variables.

sality is a puzzling question, here we address only the origin of stabilization effect following the same lines as in Sec. III B.

Insight into the cause of instability can be gained from the origin of forces of interaction. Since it is known that for a flat thin film of thickness $h$ the maximum drainage velocity is $\sim \sqrt{\mathrm{Bo}_{h}} \sigma / \mu$ (which is small in our experiments), one can neglect by the effect of drainage. Therefore, even though this physical process may trigger instability, the primary forces in the dewetting process are different from gravity. As discussed in Sec. III B, it is known that at equilibrium, hydrostatic forces tending to thin the film are counterbalanced by disjoining pressure, originating from a London-van der Waals term, an electrical double-layer term, or steric repulsion. In the range of thicknesses in our experiments, the primary contribution comes from electric double-layer forces due to dissociation of terminal silanol groups amplified by the increase in surface area of rough surface. The role of these forces in film (in)stability remains the same as for smooth substrate in the regime $\bar{h}_{\infty} \geqslant r$. However, the rough- ness itself introduces interesting aspects by modifying wetting properties of the substrate, thus eventually influencing film stability.

Even though the effect of roughness on wetting properties of the substrate is not well explored, there is a number of works advancing our understanding. Let the rough substrate to be characterized by the true and apparent contact angles, $\theta_{0}$ and $\theta^{*}$, and the roughness factor $\alpha_{s}$, the ratio of the real to apparent area. It is known since the work of Wenzel ${ }^{64}$ that the presence of roughness amplifies wetting, $\cos \theta^{*}$ $=\alpha_{s} \cos \theta_{0}$, i.e., it may either increase the contact angle if the contact angle on smooth substrate $\theta_{0}>\pi / 2$ or decreases it if $\theta_{0}<\pi / 2$. The analogous effect was shown by Bico et al. ${ }^{65}$ in the presence of air (or any other hydrophobic material) trapped in indentations between the solid and liquid phases (air pockets), which in the limit of negligible solid fraction of the surface leads to superhydrophobicity, $\theta^{*}=\pi$. However, Herminghaus $^{66}$ demonstrated that it is possible to construct a surface shape that increases the effective surface tension between the liquid film and substrate, thus increasing the contact angle and leading in the limit of a fractal surface to perfect nonwetting for any $\theta_{0}$. At the same time, the roughness may lead to improved wetting and even imbibitionspontaneous invasion by liquid-which is observed for contact angles $\theta_{0}$ below a critical one depending on the solid texture. ${ }^{67}$ Our materials do not exhibit imbibition phenomena.

Our problem imposes several challenging questions, among them: at what roughness does the film attain stability? From observations we know that a water film is stable on a 50-grit $(r=1.71 \mu \mathrm{m})$ surface compared to the one on a 400grit $(r=0.193 \mu \mathrm{m})$ one. As mentioned above, gravity drainage is negligible at least within a reasonable time, and therefore the film stability can be treated from a static standpoint. As in the above works on wetting effects of roughness, the question should be considered from a thermodynamic point of view, which however introduces excessive degrees of freedom and vagueness in developments-the work by Johnson $^{68}$ is a good account on this issue. However, following Netz and Andelman ${ }^{69}$ one can introduce a free energy as

$$
\mathcal{F}(h)=\alpha_{s} \sigma_{s l}+\alpha_{l}(h) \sigma_{l g}+P(h)+\mu h,
$$

where $\alpha_{s}$ and $\alpha_{l}$ are ratios between the actual and projected areas of the substrate surface and liquid interface, respectively, $P(h)$ is the effective wetting potential, and $\mu$ the chemical potential difference between interfaces. Minimization of (9) for a fixed average film thickness $h$ yields $\mathcal{F}^{*}(h)$, and definition of the free-energy difference, $\Delta \mathcal{F}^{*}(h)=\mathcal{F}$ $-\mathcal{F}^{*}$, yields

$$
\Delta \mathcal{F}^{*}(h)=\left\{\begin{array}{l}
\alpha_{s}\left(\sigma_{s g}-\sigma_{s l}\right)-\sigma \text { for } h \rightarrow 0, \\
0 \text { for } h \rightarrow \infty .
\end{array}\right.
$$

Therefore, the effective spreading coefficient $S_{\text {eff }}=\Delta \mathcal{F}^{*}(0)$ is always larger than the bare spreading coefficient $S$, i.e.,

$$
S_{\text {eff }}=\left(\alpha_{s}-1\right) \sigma+\alpha_{s} S, \quad S \equiv \sigma_{s g}-\sigma_{s l}-\sigma,
$$

since $\sigma>0$ and $\alpha_{s}>1$. Thus, even if the flat substrate is nonwetting $(S<0)$ roughness may give $S_{\text {eff }}>0$ and thus 


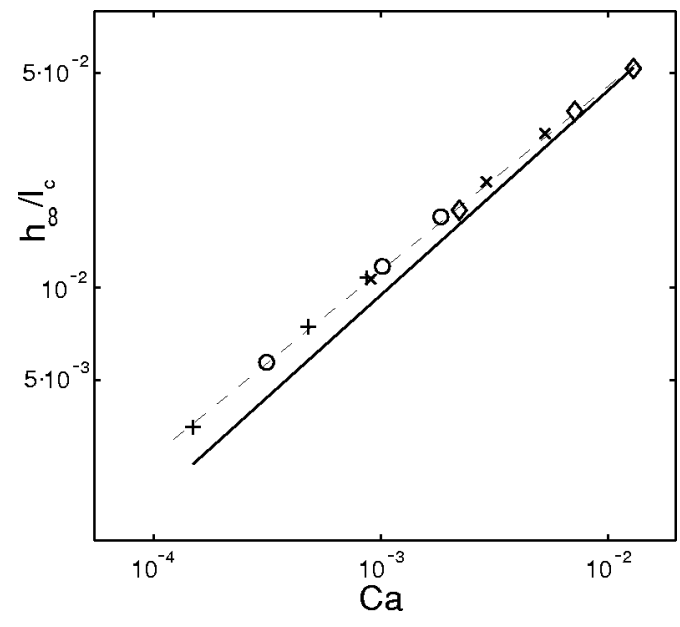

FIG. 12. Thickness of film deposited on a roughened substrate. Notations for glycerol solutions:,$+ 20 \%$ solution; $\bigcirc, 40 \%$ solution; $\times, 60 \%$ solution, $\diamond, 72 \%$ solution.

roughness-induced wetting. This thermodynamic consideration gives information not only on spreading, but also implies film stability. Our experimental observations confirmed that the increase of $\alpha_{s}$ (cf. Fig. 10), when one switches from the 400-grit to the 50-grit substrate, allows a transition from an unstable film, subject to dewetting, to a stable film, perfectly adhering the substrate. Lastly, we mention that there is no stability analysis of a film on a rough inclined substrate.

Concluding this subsection, we note that the film stability is important not only in the subsequent study of coating film thickness in Sec. IV C, but also in the engineering question of stabilization of coatings.

\section{Dynamic effect of roughness on the film thickness}

The nondimensional film thickness measured on a 50grit roughened substrate is shown in Fig. 12. The points exhibit the approximate power law behavior $\sim \mathrm{Ca}^{0.6}$, i.e., slower than that of the Landau-Levich law. From physical reasoning one can expect that there are three regimes: (1) $r / \bar{h}_{\infty} \ll 1$, so that roughness produces no observable effect, i.e., $h_{\infty} \sim C a^{2 / 3}$; (2) $r / \bar{h}_{\infty} \gg 1$, so that the effect is substantial and even the free interface is affected since the liquid fills only cavities and $h$ is independent of $\mathrm{Ca}$ (because the amount of liquid coated is determined by a static problem); and (3) an intermediate regime, $r / \bar{h}_{\infty} \sim 1$. From this logic it follows that the data of Fig. 12 are in this intermediate regime. If one takes $r$ as an average roughness, then for the data in Fig. 12, $r / \bar{h}_{\infty}=0.014-0.259$. The following discussion is devoted to understanding the reasons of the deviation from the Landau-Levich law.

In view of the substantial separation of scales (from microscopic roughness size to the macroscopic characteristic length of dip coating, $\sim l_{c}$ ), the solution of the complete problem seems to be not feasible. Therefore, it is natural to investigate the possibility of using an effective boundary condition generated by the presence of roughness as classically done in turbulent flows. ${ }^{70}$

For laminar flows the genesis of the effective boundary condition comes from Navier's idea that the slip velocity at the wall should be proportional to the shear stress $u$ $=l_{N}(\partial u / \partial y)$ [cf. Fig. 14(b) for illustration], which was later justified through kinetic theory as originating from the fraction of diffuse scattering off a flat boundary, which is usually seen on the nanoscale level. De Gennes ${ }^{71}$ exploited the Navier slip length concept in explaining an anomalous low friction for some situations, justifying it by the presence of a gaseous film near the solid/liquid interface. However, the same idea was extended onto macro- and mesoscopic level of roughness and has found numerous applications in various fields (e.g., geophysics). Einzel et al. ${ }^{72}$ suggested for the slip length $1 / l_{N}=1 / l_{N}^{0}-1 / r$, where $l_{N}^{0}$ has a classical molecular origin, while $r$ is the radius of curvature of the boundary (positive for convex and negative for concave solid boundary). While neglecting $l_{N}^{0}$, Miksis and Davis, ${ }^{23}$ in an asymptotic regime of long-wave roughness, derived the slip length being just the average roughness position $\langle\mathbf{z}\rangle$. As one can easily observe, both approaches result in a very strange effect: placing the position of effective boundary where $\langle\mathbf{z}\rangle$ $=0$ or where curvatures cancel each other, does not lead to any difference from the flat surface case. As applied to our problem this would result in no difference in terms of film thickness for both flat and undulated substrates. Also, as noted in Ref. 73, the expression of Ref. 72 in the hydrodynamic limit $l_{N}^{0} \rightarrow 0$ leads to a negligible role of roughness. The analysis by Ponomarev and Meyerovich ${ }^{73}$ as applied to the oscillating plate problem also led to a Navier-type slip model but with the sign of the slip length changing over the period of oscillations. This apparent velocity direction anisotropy where there is none in the problem was not explained in their work.

Besides the usage of an effective boundary condition, there are theories in which the effect of roughness is modeled directly. Hwang and $\mathrm{Ma}^{74}$ studied the depletion of thin liquid films due to centrifugation using lubrication equations and found enhanced retention of the film due to the presence of roughness. However, it is not clear how the lubrication approximation is applicable to the roughness with the same scales in all directions. Later, this approach incorporated the effect of disjoining pressure. ${ }^{75}$ The same approach has been utilized by Schwartz et al. ${ }^{76}$ in numerical modeling of liquid withdrawal from gravure cavities in coating operations.

\section{A simple model}

The complexity of the problem notwithstanding, there is a very simple way to test "effective" boundary conditions in a mesoscopic regime since the film thicknesses are of the order $10 \mu \mathrm{m}$. Our dynamic effects refers to the influence of roughness on a shear flow, more precisely on the drag flow. The problem is complicated by the nonuniform distribution of shear stress on the moving plate during dip coating, as shown in the moving frame in Fig. 13 in nondimensional form [obtained by numerical simulation of the dip-coating problem for a smooth solid substrate: R. Krechetnikov and G. M. Homsy (unpublished)]. The maximum of shear stress corresponds to the stagnation point at the interface, since in this region the velocity changes from zero at the stagnation 


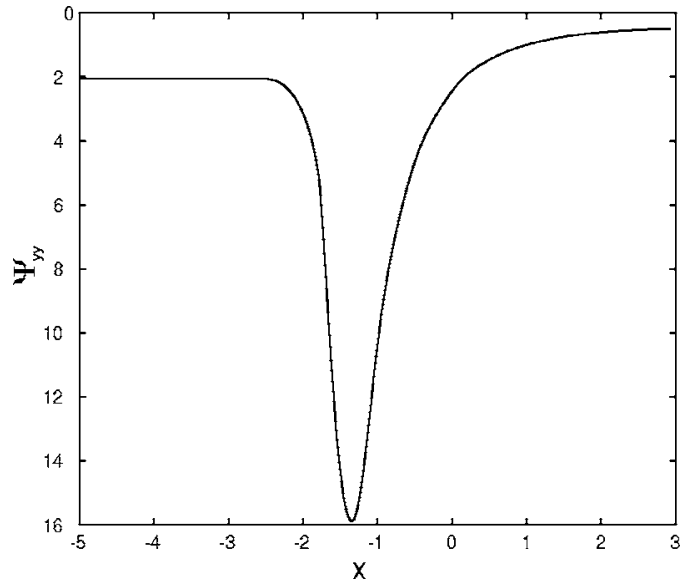

FIG. 13. Shear stress distribution at the plate for $C a=10^{-2} ;-x \gg 1$ corresponds to a thin film region, while $x \gg 1$ corresponds to the bulk.

point to -1 at the wall. The asymptotic values of shear stress in a thin film region and in the bulk are estimated as $-h / C a$ and $(4 / \pi) / x^{2}$, respectively. Nonzero shear in the thin film region is explained by a small gravity drainage.

First, we will describe different physical aspects of the problem from a phenomenological standpoint. This will help to appreciate the complexity of phenomenon and outline the objectives for a quantitative study. As a model, let us consider two situations: shear flow on a flat plate and drag flow (flow near a moving plate). For simplicity, the roughness is represented as a set of regular triangular undulations, which can be considered as an effective representation of the true roughness. In this case the flow pattern is an antisymmetric flow analyzed in Ref. 77 and sketched in Fig. 14(c). The local solution in the corner is derivable in the Stokes approximation as in Moffatt. ${ }^{78}$ For general roughness there are also numerical (cf. Ref. 79) and analytical solutions (cf. Ref.

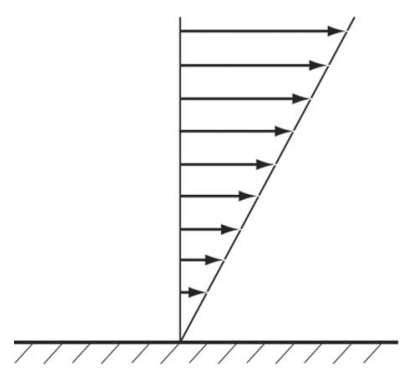

(a)

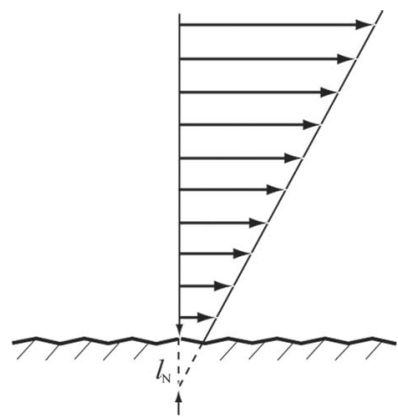

(b)

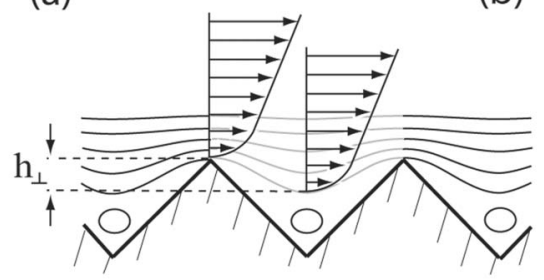

(c)

FIG. 14. Rough wall in a simple shear flow. (a) Smooth wall. (b) Rough wall. (c) Closeup.

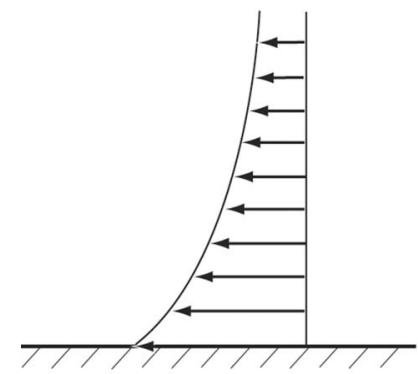

(a)

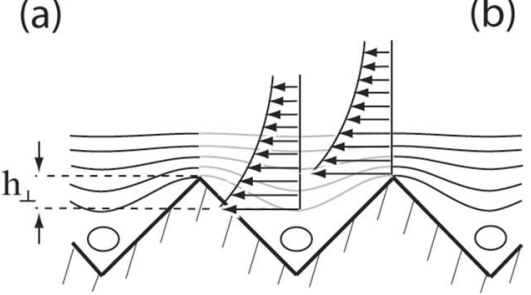

(c)

FIG. 15. Rough wall in a drag flow. (a) Smooth wall. (b) Rough wall. (c) Closeup.

80). The assumed picture is also in agreement with the spectral solutions for flow over rectangular grooves by Wang, ${ }^{81,82}$ and a solution by a Wiener-Hopf method for an idealized porous wall by Jeong. ${ }^{83}$

Next, let us consider simple shear flow as in Fig. 14(a), which can be considered as a boundary layer solution near wall corresponding to the linear profile, $u \sim y$. One can expect that the presence of roughness will enhance the production of vorticity and instead of constant vorticity as in Fig. 14(a) one ends up with a higher vorticity at the wall approaching the constant one far from the wall. This effective picture can be represented as in Fig. 14(b), i.e., by some slip length. This is justified by considering velocity profiles on the roughness scale as depicted in Fig. 14(c).

The profiles shown correspond to the zero-velocity streamline, which can be interpreted as an effective no-slip curve. On this basis, Bechert and Bartenwerfer ${ }^{84}$ introduced the notion of a protrusion height $h_{\perp}$, which designates the location of an effective plane of no-slip by which one can replace the rough boundary. As suggested by Luchini et al., ${ }^{22}$ one can define this protrusion length from an asymptotic solution far from the boundary as $u \rightarrow y+h_{\perp}$, where the origin of coordinates $y$ is chosen at the tops of the roughness. In the case of general profiles this definition is no longer applicable and one can suggest averaging the position of the zerovelocity streamline:

$$
\bar{h}_{\perp}=\frac{1}{T_{x}} \int_{0}^{T_{x}} h_{\perp}(x) \mathrm{d} x .
$$

The same line of logic can be applied to drag flows, which as seen from Figs. 15(a) and 15(b) leads to a reduced drag flow due to partial slip at the boundary. Again, the justification of this slip comes from consideration of flow structure near the wall as in Fig. 15(c). 


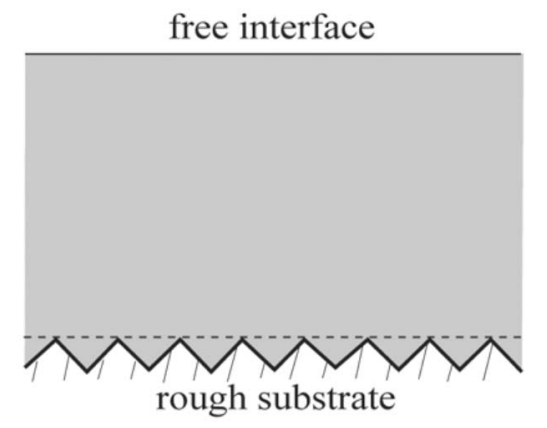

(a)

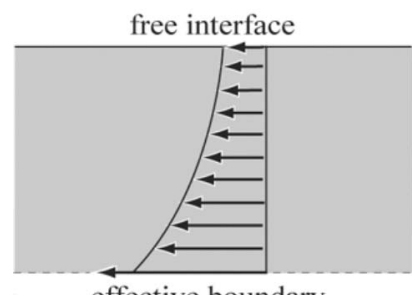

effective boundary

(b)

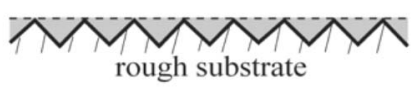

(c)
FIG. 16. Dip coating with a rough substrate. (a) True situation. (b) Effective flow with slip. (c) Liquid in grooves.

As applied to our case of a liquid film withdrawn from a bath, this phenomenology splits the complete problem [Fig. 16(a)] into the dip-coating problem with a smooth plate [Fig. 16(b) ] located at $y=-h_{\perp}$ and the additional amount of liquid in the grooves [Fig. 16(c)]. Since in experiments one can measure just an averaged mass flux, one can anticipate a nonmonotonic behavior in the dependence of film thickness on roughness size. While the described phenomenology refers to the two-dimensional picture, our problem is complicated by the three-dimensionality of the roughness distribution even though it is isotropic.

With this phenomenological justification, we consider the boundary value problem in a streamfunction form, $\psi_{y}$ $=u$, with the partial slip condition at the wall

$$
\begin{aligned}
& 0=h_{x x x}-1+C a \psi_{y y y}, \\
& y=0: \psi_{y}=-1+l \psi_{y y}, \psi=0, \\
& y=h: \psi_{y y}=0,
\end{aligned}
$$

which comes from the $x$-momentum equation $0=-p_{x}+1$ $+\operatorname{Cau}_{y y}$ and the fact that $p \sim h_{x x}$. This yields, after transforming $x \rightarrow \beta \tilde{x}, h \rightarrow h_{\infty} \tilde{h}$ (the fact that $h_{\infty} \sim \beta^{2}$ necessary for matching with the static meniscus solution is taken into account)

$\tilde{h}_{\widetilde{x} x \tilde{h}} \tilde{h}^{3}-\beta \underline{\left(\tilde{h}^{3}-1\right)}+3 \frac{C a}{\beta^{3}}(\tilde{h}-1)+3 l \frac{1-\tilde{h}^{2}}{\beta}+3 l \frac{\tilde{h}^{2} \tilde{h}_{\tilde{x} \tilde{x} x}}{\beta^{2}}=0$.

Even though the Navier slip length $l$ is small, the term $l \psi_{y y}$ can be appreciable since the shear at the wall is strongly peaked in the limit of low capillary numbers (cf. Fig. 13).
Therefore, if $l \ll C a^{2 / 3}$, one gets $\beta \sim C a^{1 / 3}$ and the LandauLevich law. If $l \gg C a^{2 / 3}$ then $\beta \sim \sqrt{l}$, resulting in a law independent of the capillary number as argued at the beginning of this subsection. Independence of $\bar{h}_{\infty}$ was originally observed by Bretherton ${ }^{17}$ and attributed to roughness effects by Chen ${ }^{24}$ for bubbles moving in capillary tube. In the intermediate regime, $l \sim C \mathrm{Ca}^{2 / 3}$, and all terms in the above general equation are important. Moreover, one does not anticipate a power law in this regime, at least in the framework of this model. Concluding, the observed power law behavior in Fig. 12 is just an approximation over the part of intermediate regime, $l$ $\sim \mathrm{Ca}^{2 / 3}$. Finding the value of the slip length $l$ is a nontrivial task, which is outside the scope of this paper. However, the knowledge of $l$ would allow a quantitative study of the problem through the integration of Eq. (14).

\section{SUMMARY AND CONCLUSIONS}

In this work we studied experimentally the surfactant and substrate roughness effects on the stability and the thickness of a coated film. The basic gravimetric procedure along with Derjaguin's idea of the lower edge effect allowed us to put the measurements on systematic grounds. In particular, the range of measurability was determined (however, no inertia and transient effects were studied). One can put both surfactant and roughness influences on the film stability in a general context. As we observed, the deposited film may exhibit either very strong dewetting instability or exceptional stability; the latter takes place for either

(1) a film substantially contaminated with surfactant on the glass substrate, or

(2) a film of pure water deposited on a rough glass substrate but with roughness size (rms) much smaller than the film thickness.

Thresholds in both roughness size and surfactant concentration were observed. In the case of coating on roughened substrates, this transition curve exhibits a universal behavior when scaled appropriately. From a general point of view the existence of the film (on a certain time scale) is dictated by the energies of film interaction with (1) the substrate, (2) gravity, and (3) itself. The first one is affected by the nature of the liquid and substrate and can be amplified by roughening the liquid-solid interface, and/or by introducing surfactants, which create double layers and other structures usually having stabilizing effects. The second is usually destabilizing since it drives the film drainage. The third one is primarily a manifestation of surface tension forces, which tend to minimize the interfacial area (as in the Plateau-Rayleigh instability). Therefore, introduction of surfactants diminishes its contribution. The net effect of different components is easily observable in experiments: introduction either of roughness or surfactants makes the film stable relative to the clean interface and smooth substrate case.

Another objective of this work was measurements of the film thickness as functions of the parameters. In particular, we performed film thickness measurements in the presence of surfactant (SDS) and determined the behavior of the thick- 
ening factor. The fast adsorption rate of this particular surfactant allowed us to show that the Marangoni effects are not responsible for the observed thickening.

The study of a rough substrate coating started with the careful and complete characterization of the roughness. The observed experimentally transition from an unstable film to a stable one with an increase of roughness size is explained theoretically by analyzing the dependence of spreading coefficient on the surface area index. The film thickness measurements demonstrate substantial deviation from the LandauLevich power law, which are explained theoretically by partial slip at the wall.

\section{ACKNOWLEDGMENTS}

The authors would like to express their gratitude to David Cinque for the help in conducting the preliminary experiments during his internship from École Polytechnique, Paris, and to Dave Bothman for help with the interferometric measurements. We also would like to acknowledge the helpful comments of anonymous referees that have led to a clearer presentation. This work was supported by the Office of Basic Energy Sciences, U. S. Department of Energy.

\section{APPENDIX: NOTES ON SANDING}

Among various artificial approaches to roughen smooth substrates (planing, sanding, bandsawing, etc.), sanding is recognized as the most advantageous processing step prior to paint application (from the standpoints of paint consumption, stability, durability and stain performance of coatings), for example, in wood industry. ${ }^{21}$ The natural question of the optimum roughness grade for the best finish performance still remains open.

Sanding, even though a quite common phenomenon in everyday life, represents a quite complicated physical process. While there are several ways to sand the surface, we have chosen the one involving simple application of sandpaper (3M Inc.). This particular sandpaper is made of silicone carbide (hardness $H \sim 1500 \mathrm{~kg} / \mathrm{mm}^{2}$, which is much harder than the substrates we studied: glass $\left(H \sim 470 \mathrm{~kg} / \mathrm{mm}^{2}\right)$, copper $\left(H \sim 92 \mathrm{~kg} / \mathrm{mm}^{2}\right)$, and stainless steel $(H$ $\left.\sim 171 \mathrm{~kg} / \mathrm{mm}^{2}\right)$. The shard-shaped grains of silicon carbide are bonded to the backing by two coats of adhesive. It is known that ceramic powders exhibit log-normal distribution, which is due to general property of the process of their production-breaking of particles-as was proved by Kolmogorov ${ }^{85}$ In the modern view, the log-normal distribution is the result of central limit theorem as applied to a variety of independent multiplicative effects (e.g., daily return rates on a stock investment). Application under known pressure (in our case $\sim 10^{6} \mathrm{~Pa}$ ) of a certain grit-number sandpaper with log-normal sizes of grains with known hardness to a substrate with also known hardness poses two natural questions:

(1) What is the distribution law for roughness sizes?

(2) What is the average roughness size?

While the first question is considered in detail in Sec. IV B, the second question represents a challenging problem in

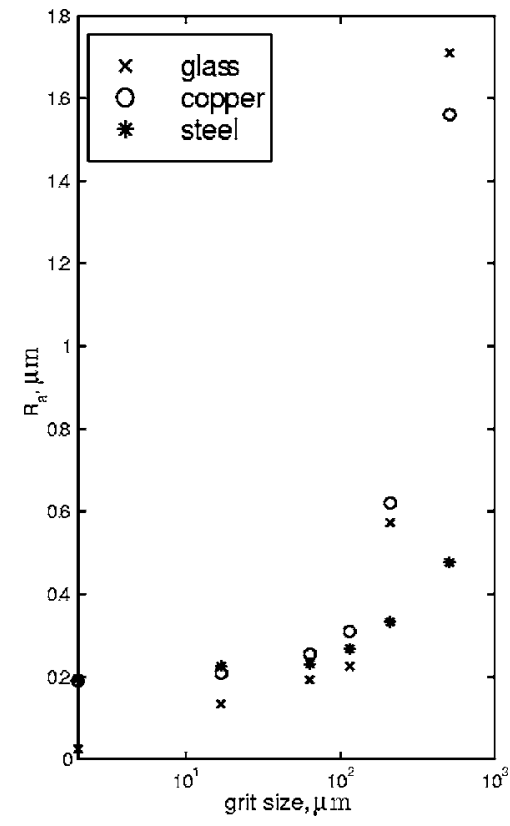

FIG. 17. Average roughness (6) measured with an optical interferometer.

abrasion theory. One can imagine sanding as a multiple process of indentations. As established by Tabor, ${ }^{86}$ the necessary condition for scratching substrate with $H_{s}$ by indenter with $H_{i}$ is $H_{i} \leqslant 1.2 H_{s}$. Since the hardness of the indenter is much higher than that of substrate, the penetration depth of a single indenter, say, of conical shape, into a substrate of hardness can be estimated from the Brinell formula $r \sim \sqrt{F_{n} / H}$, where $F_{n}$ is a normal force.

Even though this formula predicts correctly that, for example, the roughness size for copper is higher than for stainless steel, its use with glass cannot take into account the dynamic effect of scratching due to which roughness is higher for a given grit size, than for metallic substrates, as shown in Fig. 17.

To our knowledge, there is no predictive theory of dynamic abrasion, despite the high demand not only in wear studies, but also in other fields like glacier physics, where the empirical abrasion model is used under the title "sandpaper friction model." ${ }^{87}$ The above estimates with the Brinell for-

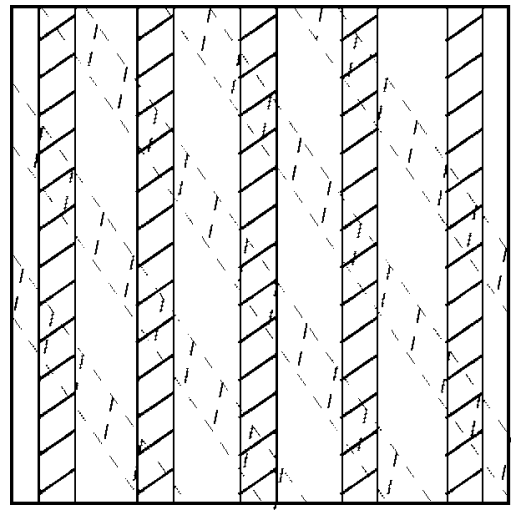

FIG. 18. Schematics of the roughening process. If the vertical grooves are the original ones, then the secondary (inclined) will lead to less mass loss (in statistical sense for large sample). 


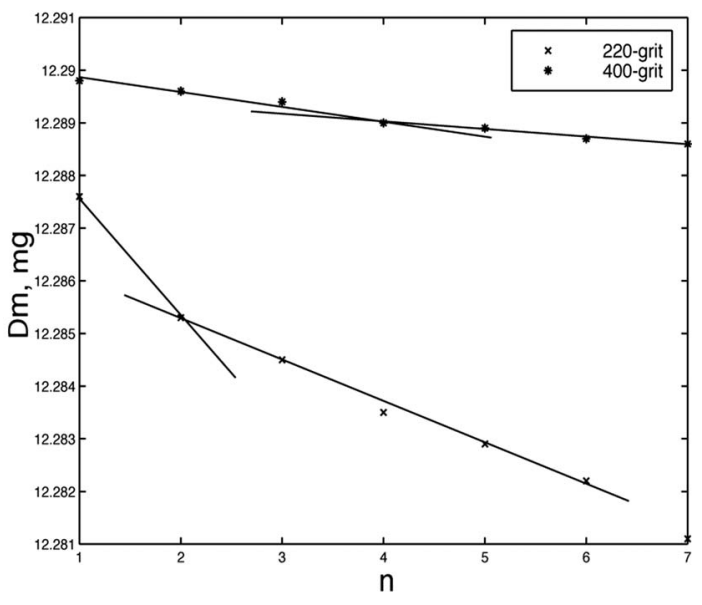

FIG. 19. Abrasion rate and gravimetric measurements of average roughness as functions of the number of sanding cycles $n$.

mula can be easily checked with a very simple gravimetric measurement of average roughness size, which is based on the observation (cf. Fig. 18) that the abrasion rate of an originally smooth substrate is higher than the asymptotic rate when the substrate is completely roughened. The transition from one regime to another defines the averaged roughness, as shown in Fig. 19.

${ }^{1}$ L. Landau and B. Levich, "Dragging of a liquid by a moving plate," Acta Physicochim. URSS 17, 42 (1942).

${ }^{2}$ D. A. White and J. A. Tallmadge, "Theory of drag out of liquids on flat plates," Chem. Eng. Sci. 20, 33 (1965).

${ }^{3}$ R. P. Spiers, C. V. Subbaraman, and W. L. Wilkinson, "Free coating of a Newtonian liquid onto a vertical surface," Chem. Eng. Sci. 29, 389 (1974).

${ }^{4}$ S. D. R. Wilson, "The drag-out problem in film coating theory," J. Eng. Math. 16, 209 (1982).

${ }^{5}$ J. A. Tallmadge, "Withdrawal of flat plates from power law fluids," AIChE J. 16, 925 (1970).

${ }^{6}$ B. M. Deryagin and S. M. Levi, Film Coating Theory (Focal, London, 1964).

${ }^{7}$ A. de Ryck and D. Quéré, "Gravity and inertia effects in plate coating," J. Colloid Interface Sci. 203, 278 (1998).

${ }^{8}$ O. O. Ramdane and D. Quéré, "Thickening factor in Marangoni coating," Langmuir 13, 2911 (1997).

${ }^{9}$ F. S. Goucher and H. Ward, "A problem of viscosity: the thickness of liquid films formed on solid surfaces under dynamic conditions," Philos. Mag. 44, 1002 (1922).

${ }^{10} \mathrm{~F}$. C. Morey, "Thickness of a liquid film adhering to surface slowly withdrawn from the liquid," J. Res. Natl. Bur. Stand. 25, 385 (1940).

${ }^{11} \mathrm{~B}$. Deryagin and A. Titievskaya, "Experimental study of liquid film thickness left on a solid wall after receeding meniscus," Dokl. Akad. Nauk SSSR 50, 307 (1945).

${ }^{12}$ A. Q. Shen, B. Gleason, G. H. McKinley, and H. A. Stone, "Fiber coating with surfactant solutions," Phys. Fluids 14, 4055 (2002).

${ }^{13}$ J. J. van Rossum, "Viscous lifting and drainage of liquids," Appl. Sci. Res., Sect. A 7, 121 (1958).

${ }^{14}$ J. P. Kizito, Y. Kamotani, and S. Ostrach, "Experimental free coating flows at high capillary and Reynolds number," Exp. Fluids 27, 235 (1999).

${ }^{15}$ D. Quéré, "Fluid coating on a fiber," Annu. Rev. Fluid Mech. 31, 347 (1999).

${ }^{16}$ P. Groenveld, "Low capillary number withdrawal," Chem. Eng. Sci. 25, 1259 (1970)

${ }^{17}$ F. P. Bretherton, "The motion of long bubbles in tubes," J. Fluid Mech. 10, 166 (1961).

${ }^{18}$ C.-W. Park, "Effects of insoluble surfactants on dip coating," J. Colloid Interface Sci. 146, 382 (1991).
${ }^{19}$ J. Ratulowski and H.-C. Chang, "Marangoni effects of trace impurities on the motion of long gas bubbles in capillaries," J. Fluid Mech. 210, 303 (1990).

${ }^{20}$ I. Golobič and K. Ferjančič, "The role of enhanced coated surface in pool boiling CHF in FC-72," Heat Mass Transfer 36, 525 (2000).

${ }^{21}$ K. Richter, W. C. Feist, and M. T. Knaebe, "The effect of surface roughness on the performance of finishes," For. Prod. J. 45, 91 (1995).

${ }^{22}$ P. Luchini, F. Manzo, and A. Pozzi, "Resistance of a grooved surface to a parallel flow and cross-flow," J. Fluid Mech. 228, 87 (1991).

${ }^{23}$ M. J. Miksis and S. H. Davis, "Slip over rough and coated surfaces," J. Fluid Mech. 273, 125 (1994).

${ }^{24}$ J.-D. Chen, "Measuring the film thickness surrounding a bubble inside a capillary," J. Colloid Interface Sci. 109, 341 (1986).

${ }^{25}$ D. A. White and J. A. Tallmadge, "A theory of withdrawal of cylinders from liquid baths," AIChE J. 12, 333 (1966).

${ }^{26}$ CRC Handbook of Chemistry and Physics, edited by D. R. Lide (CRC Press, Cleveland, OH, 2002-2003).

${ }^{27}$ M. L. Sheely, "Glycerol viscosity tables," Ind. Eng. Chem. 24, 1060 (1932).

${ }^{28}$ G. J. Gittens, "Variation of surface tension of water with temperature," J. Colloid Interface Sci. 30, 406 (1969).

${ }^{29}$ J. Fernandez, R. Krechetnikov, and G. M. Homsy, "Experimental study of a surfactant-driven fingering phenomenon in a Hele-Shaw cell," J. Fluid Mech. 527, 196 (2005).

${ }^{30}$ C.-H. Chang, N.-H. L. Wang, and E. I. Franses, "Adsorption dynamics of single and binary surfactants at the air/water interface," Colloids Surf. 62, 321 (1992).

${ }^{31} \mathrm{P}$. H. Elworthy and K. J. Mysels, "The surface tension of sodium dodecylsulfate solutions and the phase separation model of micelle formation," J. Colloid Interface Sci. 21, 331 (1966).

${ }^{32}$ K. Tajima, M. Muramatsu, and T. Sakaki, "Radiotracer studies on adsorption of surface active substance at aqueous surface. I. Accurate measurements of adsorption of tritiated sodium dodecylsulfate," Bull. Chem. Soc. Jpn. 43, 1991 (1970).

${ }^{33}$ C.-H. Chang and E. I. Franses, "Modified Langmuir-Hinshelwood kinetics for dynamic adsorption surfactants at the air/water interface," Colloids Surf. 69, 189 (1992).

${ }^{34}$ A. Sharma, "Many paths to dewetting of thin films: Anatomy and physiology of surface instability," Eur. Phys. J. E 12, 397 (2003).

${ }^{35}$ L. W. Schwartz, R. V. Roy, R. R. Eley, and S. Petrash, "Dewetting patterns in a drying liquid film," J. Colloid Interface Sci. 234, 363 (2001).

${ }^{36}$ This phenomenon was first predicted by Smoluchowski (1908) and first observed by light-scattering in experiments of Mandelstam (1913).

${ }^{37}$ T. B. Benjamin, "Wave formation in laminar flow down an inclined plane," J. Fluid Mech. 2, 554 (1957).

${ }^{38}$ C. S. Yih, "Stability of liquid flow down an inclined plane," Phys. Fluids 6, 321 (1963).

${ }^{39}$ H. J. Schulze, K. W. Stöckelhuber, and A. Wenger, "The influence of acting forces on the rupture mechanism of wetting films-nucleation or capillary waves," Colloids Surf., A 192, 61 (2001).

${ }^{40}$ A. Vrij, "Possible mechanism for the spontaneous rupture of thin, free liquid films," Discuss. Faraday Soc. 42, 23 (1966).

${ }^{41}$ M. B. Williams and S. H. Davis, "Nonlinear theory of film rupture," J. Colloid Interface Sci. 90, 220 (1982).

${ }^{42}$ G. Reiter, A. Sharma, A. Casoli, M.-O. David, R. Khanna, and P. Auroy, "Thin film instability induced by long-range forces," Langmuir 15, 2551 (1999).

${ }^{43}$ A. Sharma and R. Khanna, "Pattern formation in unstable thin liquid films under the influence of antagonistic short- and long-range forces," J. Chem. Phys. 110, 4929 (1999).

${ }^{44}$ J. A. Tallmadge and R. Stella, "Some properties of the apparent water paradox in entrainment," AIChE J. 14, 838 (1968).

${ }^{45}$ G. M. Ginley and C. J. Radke, "Influence of soluble surfactants on the flow of long bubbles through a cylindrical capillary," ACS Symp. Ser. 396, 480 (1989).

${ }^{46}$ It should be kept in mind that interpretation of St $\kappa$, as a parameter defining the rate at which the surfactant is supplied by adsorption to the interface, is not valid in general for concentrations above CMC since micelles have their own characteristic kinetic time scales. However, in the case of the SDS surfactant it is known that the characteristic time of exchange between micelles and the bulk is negligibly smaller than the rate-limiting adsorption time $t_{a}$ [see A. Patist, J. R. Kanicky, P. K. Shukla and D. O. Shah, "Importance of micellar kinetics in relation to technological processes," J. Colloid Interface Sci. 245, 1 (2002); A. Q. Shen, B. 
Gleason, G. H. McKinley and H. A. Stone, "Fiber coating with surfactant solutions," Phys. Fluids 14, 4055 (2002)].

${ }^{47}$ R. Krechetnikov and G. M. Homsy, "On a new surfactant-driven fingering phenomenon in a Hele-Shaw cell," J. Fluid Mech. 509, 103 (2004).

${ }^{48}$ Y. Zhu and S. Granick, "No-slip boundary condition switches to partial slip when fluid contains surfactant," Langmuir 18, 10058 (2002).

${ }^{49} \mathrm{~J}$. N. Israelachvili, "Measurement of the viscosity of liquids in very thin films," J. Colloid Interface Sci. 110, 263 (1986).

${ }^{50}$ B. V. Derjaguin, V. V. Karassev, V. M. Starov, and E. N. Khromova, "Study of boundary viscosity of volatile liquids by blowoff method," Colloid J. USSR 39, 584 (1977).

${ }^{51}$ H. B. Kutzner, P. F. Luckham, and J. Rennie, "Measurement of the viscoelastic properties of thin surfactant films," Faraday Discuss. 104, 9 (1996).

${ }^{52}$ A. D. Read and J. A. Kitchener, "The thickness of wetting films," Soc. Chem. Ind. (London) Monograph 25, 300 (1967).

${ }^{53}$ A. D. Read and J. A. Kitchener, "Wetting films on silica," J. Colloid Interface Sci. 30, 391 (1969).

${ }^{54}$ T. M. Squires and M. P. Brenner, "Like-charge attraction and hydrodynamic interaction," Phys. Rev. Lett. 85, 4976 (2000).

${ }^{55}$ S. H. Behrens and D. G. Grier, "The charge of glass and silica surfaces," J. Chem. Phys. 115, 6716 (2001).

${ }^{56}$ R. K. Iler, The Chemistry of Silica (Wiley, New York, 1979).

${ }^{57}$ J. F. Padday, "Cohesive properties of thin films of liquids adhearing to a solid surface," in Thin Liquid Films and Boundary Layers, Special Discussions of the Faraday Society (Academic, New York, 1970), pp. 64-88.

${ }^{58} \mathrm{~V}$. S. Mitlin, "Dewetting of solid surface: analogy with spinodal decomposition," J. Colloid Interface Sci. 156, 491 (1993).

${ }^{59}$ J. F. Gouyet, M. Rosso, and B. Sapoval, "Fractal surfaces and interfaces," in Fractal and Disordered Systems, edited by A. Bunde and S. Havlin (Springer, Berlin, 1991), pp. 229-261.

${ }^{60}$ Analogous measurements with copper and stainless steel yield pdfs with much smaller skewness and kurtosis close to 3.0, thus indicating near normal behavior. It would be beneficial to understand such a difference between glass and metallic substrates, which probably comes from the brittleness of the glass, thus differentiating the process of roughening in this case. In the comparative study of asperities in wood and aluminum sanded surfaces [T. Ohtani, C. Tanaka and H. Usuki, "Comparison of the heterogeneity of asperities in wood and aluminum sanding surfaces," Precis. Eng. 28, 58 (2004)] it was found that skewness depends on the microstructure of the substrate. A priori one can expect that if the material is not brittle, then the roughening process involves just additive independent events and results in a normal distribution, while the brittleness leads to a certain degree of multiplicativity and thus to a mixed normal and lognormal distribution. In the limit of absolute brittleness one gets to the usual picture of breakup, and therefore to log-normal distribution.

${ }^{61}$ W. J. Barun, V. Rousson, W. A. Simpson, and J. Prokop, "Parametric modelling of reaction time experiment data," Biometrics 59, 661 (2003).

${ }^{62}$ E. Limpert, W. A. Stahel, and M. Abbt, "Log-normal distributions across the sciences: Keys and clues," BioScience 51, 341 (2001).

${ }^{63}$ S. S. Shapiro and M. B. Wilk, "An analysis of variance test for normality (complete samples)," Biometrika 52, 591 (1965).

${ }^{64}$ R. N. Wenzel, "Resistance of solid surfaces to wetting by water," Ind. Eng. Chem. 28, 988 (1936).
${ }^{65}$ J. Bico, C. Tordeux, and D. Quéré, "Rough wetting," Europhys. Lett. 55, 214 (2001)

${ }^{66}$ S. Herminghaus, "Roughness-induced non-wetting," Europhys. Lett. 52, 165 (2000).

${ }^{67}$ J. Bico, C. Marzolin, and D. Quéré, "Pearl drops," Europhys. Lett. 47, 220 (1999).

${ }^{68}$ R. E. Johnson, "Conflicts between Gibbsian thermodynamics and recent treatments of interfacial energies in solid-liquid-vapor systems," J. Phys. Chem. 63, 1655 (1959).

${ }^{69}$ R. R. Netz and D. Andelman, "Roughness-induced wetting," Phys. Rev. E 55, 687 (1997).

${ }^{70}$ This idea has been exploited in a probably best studied problem with the dynamic effect of roughness-for turbulent flows [cf. H. Schlichting, Boundary Layer Theory (McGraw-Hill, New York, 1960)] in channels with rough walls, as initiated by the famous Nikuradse experiments. The curious observation in a pipe flow, for example, is a less filled profile and higher frictional resistance for the case of rough walls as opposed to the case of smooth walls. Recently the analogous study of the logarithmic "wall law" was performed for sanded surfaces by M. P. Schultz, "The relationship between frictional resistance and roughness for surfaces smoothed by sanding," ASME Trans. J. Fluids Eng. 124, 492 (2002).

${ }^{71}$ P. G. de Gennes, "On fluid/wall slippage," Langmuir 18, 3413 (2002).

${ }^{72}$ D. Einzel, P. Panzer, and M. Liu, "Boundary condition for fluid flow: Curved or rough surfaces," Phys. Rev. Lett. 64, 2269 (1990).

${ }^{73}$ I. V. Ponomarev and A. E. Meyerovich, "Surface roughness and effective stick-slip motion," Phys. Rev. E 67, 026302 (2003).

${ }^{74}$ J. H. Hwang and F. Ma, "On the flow of a thin liquid film over a rough rotating disk," J. Appl. Phys. 66, 388 (1989).

${ }^{75}$ J. S. Kim, S. Kim, and F. Ma, "Topographic effects of surface roughness on thin-film flow," J. Appl. Phys. 73, 422 (1993).

${ }^{76}$ L. W. Schwartz, P. Moussalli, P. Campbell, and R. R. Eley, "Numerical modelling of liquid withdrawal from gravure cavities in coating operations," Chem. Eng. Res. Des. 76, 22 (1998).

${ }^{77}$ C. Pozdrikidis, Introduction to Theoretical and Computational Fluid Dynamics (Oxford University Press, New York, 1997).

${ }^{78}$ H. K. Moffatt, "Viscous and resistive eddies near a sharp corner," J. Fluid Mech. 18, 1 (1964).

${ }^{79}$ C. Pozdrikidis, "The flow of a liquid film along a periodic wall," J. Fluid Mech. 188, 275 (1988).

${ }^{80}$ M. Scholle, A. Wierschem, and N. Aksel, "Creeping films with vortices over strongly undulated bottoms," Acta Mech. 168, 167 (2004).

${ }^{81}$ C. Y. Wang, "The Stokes drag due to the sliding of a smooth plate over a finned plate," Phys. Fluids 6, 2248 (1994).

${ }^{82}$ C. Y. Wang, "Flow over a surface with parallel grooves," Phys. Fluids 15, 1114 (2003).

${ }^{83}$ J.-T. Jeong, "Slip boundary condition on an idealized porous wall," Phys. Fluids 13, 1884 (2001).

${ }^{84} \mathrm{D}$. W. Bechert and M. Bartenwerfer. "The viscous flow on surfaces with longitudinal ribs," J. Fluid Mech. 209, 105 (1989).

${ }^{85}$ A. N. Kolmogorov, "On the log-normal distribution of particle sizes during break-up process," Dokl. Akad. Nauk SSSR 31, 99 (1941).

${ }^{86}$ D. Tabor, "Moh's hardness scale-a physical interpretation," Proc. Phys. Soc. London 67, 249 (1954).

${ }^{87}$ J. Schweizer and A. Iken, "The role of bed separation and friction in sliding over an undeformable bed," J. Glaciol. 28, 77 (1992). 